\title{
APPLICATIONS OF THE COVERING LEMMA FOR SEQUENCES OF MEASURES ${ }^{1}$
}

\author{
W. MITCHELL
}

\begin{abstract}
We present several applications of the covering lemma for the core model for sequences of measures, including characterizations of the large cardinal strength necessary to make the filter of closed, unbounded subsets of $\omega_{1}$ an ultrafilter or to change the cofinality of a regular cardinal, and a characterization of the minimal inner model containing an arbitrary elementary embedding.
\end{abstract}

0. Introduction. In this paper we present some results which depend on a more precise use of indiscernibles in the covering lemma for $K[\mathcal{F}]$ than was required in [Mi84a, Mi]. We will discuss the necessary background from those papers in $\S 1$ but the first two results can be given statements which do not require ideas from that paper. These are the results which were announced as (viii) and (x) on p. 230 of $[\mathbf{M i 8 4 a}]$.

0.1. THEOREM. If $\kappa$ is regular but becomes a singular strong limit cardinal of cofinality $\delta>\omega$ in a generic extension then there is an inner model in which $o(k) \geq \delta$. More generally, if $\omega<\delta=\operatorname{cf}(\kappa)<\kappa, \delta^{\omega}<\kappa$, and $\kappa$ is regular in $K(\mathcal{F})$, the core model for the maximal sequence $\mathcal{F}$ of measures, then $o(\kappa) \geq \delta$ in $K(\mathcal{F})$.

This is best possible in the sense that if $\delta$ is regular and $o(\kappa) \geq \delta$ then there is a generic extension with the same cardinals such that $\operatorname{cf}(\kappa)=\delta[\mathbf{M a 7 8}]$. If $\delta$ is replaced by $\omega$ in Theorem 0.1 then Dodd and Jensen have shown [D-J82a, D-J82b] that $o(\kappa) \geq 1$ in an inner model; in this case the converse is given by Prikry forcing [Pr71]. It is not known whether Theorem 0.1 can be strengthened by replacing the hypothesis that $\delta^{\omega}<\kappa$ with $|\kappa|>\omega_{1}$.

0.2. THEOREM $\left(\mathrm{ZF}+\mathrm{AC}_{\aleph_{0}}\right)$. If the filter $\mathrm{C}$ of closed unbounded subsets of $\aleph_{1}$ is an ultrafilter then there is an inner model with a repeat point at $\kappa$.

We will define the notion of a repeat point in $\S 1$. It is known $[\mathbf{M i 8 2}]$ that if there is a cardinal with (roughly speaking) $\kappa^{+}$repeat points then there is a generic extension in which $C$ is an ultrafilter. Both conditions have strengths strictly between $o(\kappa)=\kappa^{+}$and $o(\kappa)=\kappa^{++}$.

Theorems 0.1 and 0.2 will be proved in $\S 2$. The rest of the paper concerns the model $L^{j}$ of sets constructible from an elementary embedding $j$. More precisely, set

$$
E^{j}=\{(x, \nu): x \text { is a set of ordinals and } \nu \in j(x)\}
$$

Received by the editors August 8, 1985.

1980 Mathematics Subject Classification. Primary 03E45, 03E55, 03E35.

This work was partially supported by a grant from the National Science Foundation. 
and define $L^{j}$ to be the class of sets constructible using $E^{j}$ as a predicate. Since $L^{j}=L\left(E^{j}\right)$ is well ordered, $E^{j} \cap L^{j}$ can be coded as a class of ordinals and hence $j \uparrow L^{j}: \rightarrow L^{j(j)}$ is in $L^{j}$. Thus $L^{j}$ is the smallest model of $Z F$ containing the restriction of the elementary embedding $j$. In this paper we will discuss the structure of $L^{j}$ under the assumption that there is no inner model of $\exists \kappa o(\kappa)=\kappa^{++}$, so that the core model $K(\mathcal{F})$ exists.

There are two possibilities for $L^{j}$ which would make the model uninteresting. First, $L^{j}$ might contain little of the structure of the universe-at the worst extreme, it might just be $L^{U}$ where $U=\{x: \kappa \in j(x)\}$ is the measure associated with $j$. We show that this never happens: in fact $L^{j}$ always contains all of the essential structure of $V$ :

0.3. THEOREM. The core model $K(\mathcal{F})$ as defined in $L^{j}$ is an iterated ultrapower of the core model as defined in $V$.

The other unattractive possibility is that $j$ could code up the inessential structure of $V$ along with its essential structure-at the worst, $L^{j}$ might even be all of $V$. If $j$ is allowed to be arbitrary then this sort of thing can happen. For example, suppose that there is no model of $\exists \kappa o(\kappa)=\kappa^{++}$, so that the core model $K(\mathcal{F})$ exists, and that the first measurable cardinals $\left(\kappa_{n}: n \in \omega\right)$ in $\mathcal{F}$ are still measurable in $V$. Then for any subset $a$ of $\omega$ we can find an embedding $j$ such that $a \in L^{j}$ by letting $j$ be the iterated ultrapower which uses the measure $U_{n}$ on $\kappa_{n}$ once if $n \in a$ and $\omega$ many times otherwise. To see that this works, observe that the covering lemma implies that if $j^{U_{n}}: V \rightarrow V^{\kappa_{n}} / U_{n}$ then $j^{U_{n}} \uparrow K(\mathcal{F})$ is an iterated ultrapower of $K(\mathcal{F})$ using $\mathcal{F}\left(\kappa_{n}, 0\right)=U_{n} \cap K(\mathcal{F})$ finitely many times, so $n \in a$ iff the iterated ultrapower $j \uparrow K(\mathcal{F})$ of $K(\mathcal{F})$ uses $\mathcal{F}\left(\kappa_{n}, 0\right)$ finitely many times. But by Theorem $0.3 K(\mathcal{F})^{L^{j}}$ is an iterated ultrapower of $K(\mathcal{F})$, and since $U_{n} \cap L^{j} \in L^{j}$ for all $n \in \omega$ the relevant part of $K(\mathcal{F})^{L^{j}}$ is the same as $K(\mathcal{F})$, so $n \in a$ iff $L^{j} \vDash(j \uparrow K(\mathcal{F})$ uses $\mathcal{F}\left(\kappa_{n}, 0\right)$ only finitely many times) and hence $a \in L^{j}$.

Our final result is that this does not happen if $j$ is simple enough:

0.4. THEOREM. Suppose that $j: V \rightarrow V^{\kappa} / U$ is the canonical embedding, where $U$ is a normal measure on $\kappa$. Then $L^{j} \vDash V=K(\mathcal{F})$.

It is not known whether Theorems 0.3 and 0.4 extend to models containing larger cardinals. If they do then it might be possible to consider using the models $L^{j}$ as an alternate approach to obtaining inner models. It should be noted, however, that the arguments of this paper depend heavily on the previous existence of the inner model theory for sequences of measures and it seems likely that if an extension of these arguments is possible then it will depend even more heavily on a pre existing inner model theory for the cardinals concerned.

The next section will deal with the extensions of the covering lemma for sequences of measures. We will begin with a very brief outline of the proof of the covering lemma and then use these ideas in an analysis of the indiscernibles arising from the lemma. $\S 2$ will then use these results to prove Theorems 0.1 and 0.2 . $\S 3$ begins the discussion of Theorems 0.3 and 0.4 ; it contains the proof of Theorem 0.3 and also of a simple case of Theorem 0.4 which contains the basic idea of the general result without the complications of fine structure and indiscernibles. The proof of Theorem 0.4 is given in $\S 4$ except for one lemma which is proved in $\S 5$. 
1. The covering lemma. In [Mi] the covering lemma, Lemma 6.3, was stated only in a limited form which made no reference to the indiscernibles used in a covering. The proof given there, however, readily yields the result we will need. We will outline the proof from [Mi] and then extract the results we need. For more details the reader should, of course, go to $[\mathbf{M i 8 4 a}]$ and $[\mathbf{M i}]$. The definition of $K(\mathcal{F})$, for an arbitrary sequence $\mathcal{F}$, is given in [Mi84a]. The most important part of $[\mathbf{M i}]$ from our point of view will be the proof of the covering lemma given in $\S 5$ of that paper, although the material from $\S \S 6$ and 7 will also be of use. The most complicated part of that paper, the fine structure material in $\S 4$, will not be used directly here.

The letters $\mathcal{F}$ and $\mathcal{G}$ always denote coherent sequences of measures as in [Mi74]. An $\mathcal{F} \uparrow \kappa$-mouse is a transitive set $m=J_{\eta}^{\mathcal{G}}$ such that

(i) $\mathcal{G} \uparrow \kappa=\mathcal{F} \kappa$ and $m \vDash$ " $\mathcal{G}$ is a coherent sequence of measures",

(ii) every iterated ultrapower of $m$ is well founded, and

(iii) there is a function $h^{m}, \Sigma_{1}$ definable over $m$ (from a parameter $p$ ) such that $m=h^{m \prime \prime} \kappa$.

$K(\mathcal{F})$ is the smallest set which contains $\mathcal{F}$ and all $\mathcal{\lceil}\langle\nu$-mice for all ordinals $\nu$. In this paper $₹$ will always denote the maximal sequence of measures as defined in [Mi] under the assumption that there is no inner model of $\exists \kappa o(\kappa)=\kappa^{++}$. This sequence is maximal in the sense that if $U$ is any measure on $P(\nu) \cap K(\mathcal{F})$ for some $\nu$ then $U \cap K(\mathcal{F})$ is in $₹$ and in fact if $i: K(\mathcal{F}) \rightarrow M$ is any elementary embedding into a well-founded class $M$ then $i$ is an iterated ultrapower by measures in $\mathcal{F}$.

The proof of the covering lemma starts with a cardinal $\kappa$ which is regular in $K(\mathcal{F})$ but singular in the real world, and uses a set $N \prec H_{\kappa^{+}}$such that ${ }^{\omega} N \subset N$, $|N|<\kappa$, and $N \cap \kappa$ is cofinal in $\kappa$. Our analysis will deal with arbitrary sets $N$. We will always use $N$ to denote an elementary substructure of $H_{\lambda}$ for some sufficiently large $\lambda$ (which will not generally be explicitly mentioned) such that ${ }^{\omega} N \subset N$. We will frequently put additional conditions on $N$, notably that $N$ is closed under longer than countable sequences and that $N$ contains various sets of current interest. Let $\pi: M \cong N$ be the transitive collapse of $N$. If $\pi$ is the identity, that is, if $N$ is transitive, then there is nothing more to say, so we assume that $N$ is not transitive and hence $\pi(\xi)>\xi$ for some $\xi<\kappa$. If every $\pi^{-1}(\xi) \uparrow \xi$-mouse were in $M$ then it would be possible to extend the map $\pi$ to all of $K(\mathcal{F})$ so that the measure induced on the critical point of $\pi$ is not in $K(₹)$, contradicting the maximality of $\mathcal{F}$. Thus there is a $\pi^{-1}(\mathcal{F}) \uparrow \xi$-mouse $n=J_{\eta}^{\mathcal{G}}$ which is not in $M$. We now use iterated ultrapowers to match the sequences $\mathcal{G}$ and $\pi^{-1}(\mathcal{F})$. It turns out that only $\mathcal{G}$ needs to be moved in the process, so we obtain an iterated ultrapower $i: J_{\eta}^{\mathcal{G}} \rightarrow J_{\eta^{\prime}}^{\mathcal{G}^{\prime}}$ such that $\mathcal{G}^{\prime} \uparrow \pi^{-1}(\kappa)=\mathcal{F} \uparrow \pi^{-1}(\kappa)$. The iteration points of $i$ form a system of indiscernibles for $M$, and the images of these indiscernibles under $\pi$ form a system $C^{N}$ of indiscernibles for $N$. The universal $\Sigma_{1}$ function $h^{m}$ for $m$ maps to a function $h^{\prime}$ over $J_{\eta}^{\mathcal{G}}$ such that $M \cap H_{\pi^{-1}(\kappa)}$ is contained in the smallest set $h^{m \prime \prime}(\xi ; C)$ containing $\xi$, closed under $h^{\prime}$, and containing the indiscernibles for $(\alpha, \beta)$ whenever it contains $(\alpha, \beta)$. This in turn is mapped by $\pi$ to a function $h^{N}$ such that $N \cap H_{\kappa} \cap K(\mathcal{F})$ is contained in $h^{N \prime \prime}(\pi(\xi) ; C)$. Finally, an inductive argument can be used to reduce the ordinal $\pi(\xi)$ to some ordinal $\xi^{\prime}<|N|^{+}$.

We now list some basic facts about the system $C^{N}$ of indiscernibles and the function $h^{N}$; these facts all fall out from the construction outlined above. 
1.1. Facts. (i) Let $H$ be the set of functions $\left\{\lambda x h^{N}(n, x): n \in \omega\right\}$. Then $H$ contains the rudimentary functions, the order function $o^{\mathcal{F}}$, the coherence function $C$ for $\mathcal{F}$, and the characteristic function of $\mathcal{F}$. In addition it is closed under composition.

(ii) The function $h^{N}$ is in $K(\mathcal{F})$.

Here (and elsewhere) we indifferently write $h^{N}(y, z)$ and $h^{N}(\langle x, y\rangle)$. We write $h^{N \prime \prime} x$ for $\left\{h(z): z \in^{<\omega}(z \cup \xi)\right\}$; it is this closure of a set $x$ under $h$ with which we will be primarily concerned, rather than with $h^{N}$ itself.

We say that $\vec{c}$ is a support if $\vec{c}$ is a finite increasing sequence such that for each $c \in \vec{c}, c \in C^{N}(\alpha, \beta)$ for some pair $(\alpha, \beta)$ in $h^{\prime \prime}(\vec{c} \cap c)$.

(iii) (a) $N \cap K(\mathcal{F})=\bigcup\left\{h^{\prime \prime}(\vec{c}): \vec{c}\right.$ is a support $\}$.

(b) If $\vec{c}$ is a support and $x \in h^{\prime \prime}(\vec{c} \cup \operatorname{rk}(x)+1)$ then $x \in h^{\prime \prime}(\operatorname{rk}(x)+1)$; and if $x \in h^{\prime \prime} \vec{c}$ then $x \in h^{\prime \prime}(\vec{c} \cap(\operatorname{rk}(x)+1))$. Here $\operatorname{rk}(x)$ is the rank of $x$ under the well-founded relation ' $\in$ '.

(c) If $c \in C(\alpha, \beta)$ and $\gamma<o(c)$ then there is $\beta^{\prime} \in \beta \cap h^{N \prime \prime} c$ such that $\gamma=$ $C\left(\alpha, \beta^{\prime}, \beta\right)(c)$, where $C$ is the coherence function for $\mathcal{F}$ : that is, $\lambda \nu<\kappa C\left(\alpha, \beta^{\prime}, \beta\right)(\nu)$ is the least function $f$ in $K(\mathcal{F})$ such that $[f]_{\mathcal{F}(\alpha, \beta)}=\beta^{\prime}$.

(iv) (a) If $c \in \mathcal{C}(\alpha, \beta)$ and $x \in h^{N \prime \prime} c$ then $c \in x$ iff $x \in \mathcal{F}(\alpha, \beta)$.

(b) If $c, c^{\prime} \in C(\alpha, \beta)$ and $x \in h^{N \prime \prime}\left(\inf \left(c, c^{\prime}\right)\right)$ then $c \in x$ iff $c^{\prime} \in x$.

Note that by (iii), (iv)(a) applies whenever $x \in h^{\prime \prime}(c \cup \vec{c})$ and $\vec{c} \cap[c, \alpha)=\varnothing$, and similarly for (ii)(b).

So far everything is implicit in Lemma 6.3 of [Mi], or at least in the proof of that lemma. In particular nothing we have said so far applies beyond $N$. In the Dodd-Jensen core model $K$ for $L^{\mu}$, the set $C^{N}$ of indiscernibles is a Prikry sequence for $L^{\mu}$ and is unique except for finitely many changes [D-J82a, D-J82b]. In our case it is also a set of indiscernibles for $K(\mathcal{F})$ and is close to being unique, though the situation is more complicated than it is for $L^{\mu}$. One important difference is that in the core model for a single measure there is a single Prikry sequence $C$ which will work for any set $N$, but there need not be any such universal system of indiscernibles for $K(\mathcal{F})$ (see [Mi84b]).

If $c$ is an indiscernible in $C^{N}$ then we write $\alpha^{N}(c)$ and $\beta^{N}(c)$ for the unique pair $(\alpha, \beta) \in h^{N \prime \prime} c$ such that $c \in C^{N}(\alpha, \beta)$. Then whenever $c \in C^{N}\left(\alpha^{\prime}, \beta^{\prime}\right)$ for $\alpha^{\prime} \neq \alpha$ we have $\alpha^{\prime} \in C^{N}\left(\alpha, \beta^{\prime \prime}\right)$ for some $\beta^{\prime \prime}>\beta$ such that $\beta^{\prime}=C\left(\alpha, \beta, \beta^{\prime \prime}\right)\left(\alpha^{\prime}\right)$.

1.2. LEMMA. Suppose that $N$ and $N^{\prime}$ are as in the statement of the covering lemma. Then

(i) $\left\{c \in N \cap N^{\prime}: c \in C^{N} \backslash C^{N^{\prime}}\right\}$ is finite.

(ii) $\left\{\alpha: \exists c \in C^{N} \cap C^{N^{\prime}} \alpha=\alpha^{N}(c) \neq \alpha^{N^{\prime}}(c)\right\}$ is finite.

(iii) Suppose that $c_{n} \in \mathcal{C}^{N}\left(\alpha_{n}, \beta_{n}\right)$, where $c_{n}<\alpha_{n} \leq c_{n+1}$ for each $n \in \omega$. If $g$ is any function in $K(\mathcal{F})$ then except for finitely many $n$ we have $c_{n} \in x$ iff $x \in \mathcal{F}\left(\alpha_{n}, \beta_{n}\right)$ for all $x \in g^{\prime \prime}\left(c_{n} \cup\left\{\alpha_{n}\right\}\right)$.

(iv) $\left\{\alpha: \exists c \in C^{N} \cap C^{N^{\prime}}: \alpha=\alpha^{N}(c)=\alpha^{N^{\prime}}(c)\right.$ and $\left.\beta^{N}(c) \neq \beta^{N^{\prime}}(c)\right\}$ is finite.

Proof. (i) Suppose that $c_{n} \in C^{N} \backslash C^{N^{\prime}}$ for each $n \in \omega$. Then $c_{n} \in h^{N^{\prime \prime \prime}}\left(c_{n}\right)$ for each $n \in \omega$. Since $N$ is an elementary substructure of $H_{\lambda^{+}}$for sufficiently large $\lambda$, $N \vDash \exists g \in K(\mathcal{F}) \forall n \in \omega c_{n} \in g^{\prime \prime} c_{n}$ (recall that ${ }^{\omega} N \subset N$, so $\left(c_{n}: n \in \omega\right.$ ) is in $N$ ). Let $\vec{c}$ be a support for such a $g$. Then $c_{n} \in h^{\prime \prime}\left(\vec{c} \cup c_{n}\right)$ for each $n$ in $\omega$. It follows 
from Fact 1.1(i), (iii) that $c_{n} \in h^{\prime \prime}\left(\left(\vec{c} \cap c_{n}+1\right) \cup c_{n}\right)$ and except for the finitely many $c_{n}$ 's which are in $\vec{c}$ it follows that $c_{n} \in h^{\prime \prime} c_{n}$, which contradicts Fact 1.1(iv).

(ii) If part (ii) fails then by symmetry we can assume that there is a sequence $\left(c_{n}: n \in \omega\right)$ such that $c_{n}<\alpha^{N^{\prime}}\left(c_{n}\right)<\alpha^{N}\left(c_{n}\right)<c_{n+1}$ for each $n \in \omega$. It follows that there is a function $g$ in $K(\mp)$ such that $g^{\prime \prime} c_{n} \cap\left[c_{n}, \alpha^{N}\left(c_{n}\right)\right) \neq \varnothing$ for each $n$, and hence that there is such a function in $N$. This is impossible except for the finitely many $n$ 's such that the support of $g$ has nonempty intersection with $\left[c_{n}, \alpha^{N}\left(c_{n}\right)\right)$.

(iii) If part (iii) fails then there is a function $g$ in $N$ witnessing its failure, but this is impossible except for the finitely many $n$ 's such that the support of $g$ has nonempty intersection with the interval $\left[c_{n}, \alpha_{n}\right)$.

(iv) If part (iv) fails then let $c_{n} \in C^{N}\left(\alpha_{n}, \beta_{n}\right) \cap C^{N^{\prime}}\left(\alpha_{n}, \beta_{n}^{\prime}\right)$, where $c_{n}<\alpha_{n}<$ $c_{n+1}$ and $\beta_{n} \neq \beta_{n}^{\prime}$ for each $n$ in $\omega$. Then the sequence $\left(\left(c_{n}, \alpha_{n}\right): n \in \omega\right)$ satisfies (iii) for both of the sequences $\left(\beta_{n}: n \in \omega\right)$ and $\left(\beta_{n}^{\prime}: n \in \omega\right)$, and we have $\beta_{n}^{\prime} \in h^{N^{\prime \prime \prime}}\left(c_{n}\right)$ for each $n$. Then $N$ satisfies that there is a function $g \in K(\mathcal{F})$ and ordinals $\beta_{n}^{\prime \prime} \in g^{\prime \prime} c_{n}$ such that $\beta_{n}^{\prime \prime} \neq \beta_{n}$ and (iii) is satisfied by the sequence $\left(\beta_{n}^{\prime \prime}: \nu \in \omega\right)$. But then the least $x$ in the ordering of $K(\mathcal{F})$ such that $x \in \mathcal{F}\left(\alpha_{n}, \beta_{n}\right) \backslash \mathcal{F}\left(\alpha_{n}, \beta_{n}^{\prime \prime}\right)$ is in $h^{N \prime \prime}\left(c_{n}\right)$ except for those finitely many $n$ 's such that the support of $g$ has nonempty intersection with the interval $\left[c_{n}, \alpha_{n}\right)$ and thus the truth of (iii) for $x$ and the sequence of $\beta_{n}$ 's implies its failure for the $\beta^{\prime \prime}$ sequence.

Lemma 1.2 leaves only one major question open concerning the absoluteness of $C^{N}$ : if $c \in C(\alpha, \beta)$ where $\alpha$ is one of the finitely many places at which Lemma 1.2(iv) fails then what can we say about $c$ in $C^{N^{\prime}}$ ? The answer to this is not known in general, but we can answer it under stronger assumptions.

1.3. Definition. $\beta$ is a (weak) repeat point for $\mathcal{F}$ at $\alpha$ if for each set $A$ in $\mathcal{F}(\alpha, \beta)$ there is a $\beta^{\prime}<\beta$ such that $A \in \mathcal{F}\left(\alpha, \beta^{\prime}\right)$.

If there are no repeat points in $K(\mathcal{F})$ then for each $\beta<o(\alpha)$ there is a set $A$, definable from $\beta$ in $K(\mathcal{F})$, such that $A \in \mathcal{F}(\alpha, \beta)$ but $A \notin \mathcal{F}\left(\alpha, \beta^{\prime}\right)$ for $\beta^{\prime} \neq \beta$ : let $A^{\prime}$ be least such that $A^{\prime} \in \mathcal{F}(\alpha, \beta)$ but $A^{\prime} \notin \mathcal{F}\left(\alpha, \beta^{\prime}\right)$ for $\beta^{\prime}<\beta$, and set $A=\left\{\nu \in A^{\prime}: \forall \gamma<o(\nu) A^{\prime} \cap \nu \notin \mathcal{F}(\nu, \gamma)\right\}$. This enables us to fix with some reliability which measures an indiscernible belongs to.

1.4. Lemma. Suppose that $N,\left(c_{n}: n \in \omega\right),\left(\alpha_{n}: n \in \omega\right)$, and $\left(\beta_{n}: n \in \omega\right)$ are as in Lemma 1.4(iii) and that there are no repeat points in $K(\mathcal{F})$. Then for any $N^{\prime} \subset N$ we have that $c_{n} \in C^{N^{\prime}}\left(\alpha_{n}, \beta_{n}\right)$ except for finitely many $n$.

Proof. By Lemma 1.3(iv), $\left\{\alpha^{N}\left(c_{n}\right): c_{n} \notin C^{N^{\prime}}\left(\alpha_{n}, \beta_{n}\right)\right\}$ is finite, so we can assume that $\alpha^{N}\left(c_{n}\right)=\alpha$ is constant. It follows that $\left\{n: \alpha^{N^{\prime}}\left(c_{n}\right)<\bigcup_{n} c_{n}\right\}$ is finite so we can assume that $\alpha^{N}\left(c_{n}\right)=\alpha^{\prime}$ is also constant. Then $\alpha^{N}\left(\alpha_{n}\right)=\alpha$ and $\alpha^{N^{\prime}}\left(\alpha_{n}\right)=\alpha^{\prime}$ as well. Let $c_{n} \in C^{N^{\prime}}\left(\alpha^{\prime}, \gamma_{n}\right)$ and $\alpha_{n} \in C^{N^{\prime}}\left(\alpha^{\prime}, \lambda_{n}\right)$. We claim that $\gamma_{n}<\lambda_{n}$ for all but finitely many n's. Otherwise we have, using the fact that there are no repeat points, sets $A_{n} \in h^{N^{\prime \prime \prime}}\left(c_{n}\right)$ for each $n$ such that $c_{n} \in A_{n}$ but $A_{n} \notin \mathcal{F}\left(\alpha_{n}, \beta\right)$ for any $\beta<o\left(\alpha_{n}\right)$, contradicting Lemma 1.4(iii). But now it follows that for all but finitely many $n$ we have $c_{n} \in C^{N^{\prime}}\left(\alpha_{n}, C\left(\alpha^{\prime}, \gamma_{n}, \lambda_{n}\right)\left(\alpha_{n}\right)\right)$ and it is immediate from the remark above that this implies that $\beta_{n}=C\left(\alpha^{\prime}, \gamma_{n}, \lambda_{n}\right)\left(\alpha_{n}\right)$.

1.5. LEMMA. Suppose that $N^{\prime} \supset N \cup\left\{h^{N}\right\}$, and any of the following conditions hold:

(i) There is no weak repeat point for $₹$ at $\alpha$. 
(ii) ${ }^{c f(}(\alpha) N \subset N$.

(iii) For all $c \in \bigcup_{\beta<o(\alpha)} C(\alpha, \beta)$ there are $c^{\prime} \geq c$ and $\beta^{\prime} \geq \beta(c)$ such that $c^{\prime} \in$ $C^{N}\left(\alpha, \beta^{\prime}\right) \cap C^{N^{\prime}}\left(\alpha, \beta^{\prime}\right)$.

Then the set of $c$ such that $c \in C^{N}(\alpha, \beta) \backslash C^{N^{\prime}}(\alpha, \beta)$ is bounded in $\alpha$. (For clause (ii) we have a more general result: if $D$ is an unbounded subset of $\alpha, c \in C^{N}\left(\alpha, \beta_{\nu}\right)$ for each $c \in D$, and $D$ and $\left(\beta_{c}: c \in D\right)$ are in $N$ then $\left\{c \in D: c \notin C^{N^{\prime}}\left(\alpha, \beta_{c}\right)\right\}$ is bounded in $\alpha$.)

ProOF. (i) Let $\vec{c}$ be the support of $h^{N}$ in $N^{\prime}$ and let $c$ be any indiscernible in $C^{N^{\prime}}(\alpha, \beta)$ large enough that $\vec{c} \cap[c, \alpha)=\varnothing$. Since there are no repeat points for $\mathcal{F}$ at $\alpha$, there is a set $A_{\beta}$, definable from $\beta$ in $K(\mathcal{F})$, such that $A_{\beta} \in \mathcal{F}(\alpha, \beta)$ but $A_{\beta} \notin \mathcal{F}\left(\alpha, \beta^{\prime}\right)$ for any $\beta^{\prime} \neq \beta$. Then $A_{\beta} \in h^{N \prime \prime} c$ so $c \in A_{\beta}$. But $\beta \in h^{N^{\prime \prime}} c$ and $c$ is an indiscernible for $\alpha$ in $N^{\prime}$, so $c \in A_{\beta}$ implies $c \in C(\alpha, \beta)$.

(ii) We prove the more general result stated at the end; clause (ii) follows immediately. If the conclusion fails then there is a sequence $\left(x_{c}: c \in D\right)$ and a function $g \in K(\mathcal{F})$ such that $x_{c} \in g^{\prime \prime} c$ for all $c \in D$ and such that for cofinally many $c$ we have $x_{c} \in \mathcal{F}\left(\alpha, \beta_{c}\right)$ but $c \notin x_{c}$. Since $D$ and $\left(\beta_{c}: c \in D\right)$ are in $N$, it follows that there is such a function $g$ and sequence $\left(x_{c}: c \in D\right)$ in $N$, but then we have $x_{c} \in \mathcal{F}\left(\alpha, \beta_{c}\right)$ iff $c \in x_{c}$ for all $c$ larger than the largest member of the support of $g$ below $\alpha$.

(iii) Suppose that the conclusion fails. Then we can pick sequences $\left(\nu_{n}: n \in\right.$ $\omega)$ and $\left(\xi_{n}: n \in \omega\right)$ such that for each $n$ we have $\nu_{n}<\xi_{n}<\nu_{n+1}, \nu_{n} \in D$, $\nu_{n} \notin C^{N^{\prime}}\left(\alpha, \beta_{\nu_{n}}\right), \xi_{n} \in C^{N}\left(\alpha, \lambda_{n}\right) \cap C^{N^{\prime}}\left(\alpha, \lambda_{n}\right), \lambda_{n} \geq \beta_{\nu_{n}}$, and if $\lambda_{n}>\beta_{\nu_{n}}$ then $\nu_{n} \in \mathcal{C}^{N^{\prime}}\left(\alpha, \gamma_{n}\right)$ for some $\gamma_{n}<\beta_{\nu_{n}}$.

Suppose for the moment that $\lambda_{n}=\beta_{\nu_{n}}$ for infinitely many $n$. Then there are sets $\left(X_{n}: n \in \omega\right)$ such that $\nu_{n} \in X_{n}$ but $\xi_{n} \notin X_{n}$ and $X_{n} \in h^{N^{\prime \prime \prime}}\left(\nu_{n}\right)$. It follows that $N$ satisfies that there is a function $g$ in $K(\mathcal{F})$ and sets $X_{n}$ with the same properties, but this is impossible except for those $n$ 's such that the support of $g$ has nonempty intersection with the interval $\left[\nu_{n}, \xi_{n}\right]$.

Now suppose that $\beta_{\nu_{n}}<\lambda_{n}$ for each $n$. We can assume that the sequences were chosen so that the support of $h^{N}$ in $N^{\prime}$ has empty intersection with the interval $\left[\nu_{0}, \alpha\right)$. It follows that both $\gamma_{n}$ and $\beta_{\nu_{n}}$ are in $h^{N^{\prime \prime \prime}}\left(\nu_{n}\right)$, and hence so is the least set $X_{n} \in \mathcal{F}\left(\alpha, \gamma_{n}\right) \backslash \mathcal{F}\left(\alpha, \beta_{\nu_{n}}\right)$. Also, we must have

$$
C\left(\alpha, \gamma_{n}, \beta_{\xi_{n}}\right)\left(\xi_{n}\right) \neq C\left(\alpha, \beta_{\nu_{n}}, \beta_{\xi_{n}}\right)\left(\xi_{n}\right) .
$$

Thus $X_{n}$ is a set such that $\nu_{n} \in X_{n}$ and $X_{n} \notin \mathcal{F}\left(\xi_{n}, \beta_{\nu_{n}}, \beta_{\xi_{n}}\right)\left(\xi_{n}\right)$, and there is a function $g \in K(\mp)$ such that $X_{n} \in g^{\prime \prime} \nu_{n}$ for each $n$, contradicting Lemma 1.2(iii).

We finish up this section with a look at sequences of indiscernibles for $\alpha$ which are like those in (ii) above but are not cofinal in $\alpha$.

1.6. Lemma. Suppose that $\left(c_{\nu}: \nu<\delta\right) \in N$ is an increasing sequence, that $c=\bigcup_{\nu} c_{\nu}<\alpha$, and that $c_{\nu} \in C^{N}\left(\alpha, \beta_{\nu}\right)$ for each $\nu$ where $\left(\beta_{\nu}: \nu<\delta\right) \in N$ is a nondecreasing sequence. Then $c \in C^{N}(\alpha, \beta)$ for some $\beta$ such that $\beta>\beta_{\nu}$ for all $\nu<\delta$.

PROOF. $c$ must be in $N$ since the sequence $\left(c_{\nu}: \nu<\delta\right)$ is in $N$, and $c$ must be in $c^{N}(\alpha, \beta)$ for some $\beta$ since $h^{N \prime \prime} c \cap[c, \alpha)=\varnothing$. Suppose that $\beta \leq \beta \nu$ for some $\nu$ and 
assume that $\beta_{\nu}<\beta_{\xi}$ for $\xi>\nu$ (the case of equality is similar). Define $U$ to be the set of $x \subset c$ such that for every sufficiently large $\xi, x \cap c_{\xi} \in \mathcal{F}\left(c_{\xi}, C\left(\alpha, \beta_{\nu}, \beta_{\xi}\right)\left(c_{\xi}\right)\right)$. By the argument of Lemma $1.4(\mathrm{ii}), U$ is a measure on $c$ in $K(\mathcal{F})$, and since the sequences $\left(c_{\nu}: \nu<\delta\right)$ and $\left(\beta_{\nu}: \nu<\delta\right)$ are in $N, U$ is in $N$. It follows by Fact 1.1(iii)(c) that $U=\mathcal{F}(c, C(\alpha, \gamma, \beta)(c))$ for some $\gamma \in \beta \cap h^{N \prime \prime} c$. Since both $\gamma$ and $\beta_{\nu}$ are in $h^{N \prime \prime} c$, there is a set $A$ in $h^{N \prime \prime} c$ such that $A \in \mathcal{F}\left(\alpha, \beta_{\nu}\right)$ but $A \notin \mathcal{F}(\alpha, \gamma)$. Thus $A \in U$ by the definition of $U$, but $A \notin \mathcal{F}(\alpha, \gamma)$ and hence $A \notin \mathcal{F}(\alpha, C(\alpha, \gamma, \beta)(c))$. This contradiction establishes the lemma.

\section{Proofs of Theorems 0.1 and 0.2 .}

PROOF OF THEOREM 0.1. We begin with a proof of the general form of the theorem: We are given a regular cardinal $\kappa$ of $K(\mathcal{F})$ such that $\omega<\delta=\operatorname{cf}(\kappa)<\kappa$ and $\delta^{\omega}<\kappa$. Pick $N$ so that $\delta \cup\{\delta, \kappa\} \subset N$ and $|N|<\kappa$. Since ${ }^{\omega} N \subset N, N$ satisfies that $\operatorname{cf}(\kappa)=\delta$, and $\delta \subset N, N \cap \kappa$ is countably closed and unbounded. Then $\{\nu: \exists \alpha \geq \kappa \exists \beta<o(\alpha) \nu \in C(\alpha, \beta)\}$ contains a countably closed, unbounded set $E$ : otherwise there would be a stationary set $X$ of ordinals $c$ such that $h^{N \prime \prime} c \cap[c, \kappa) \neq \varnothing$, but then there would be a stationary subset $X^{\prime}$ of $X$ and a fixed $\gamma<\kappa$ such that $h^{N \prime \prime} \gamma \cap[c, \kappa) \neq \varnothing$ for all $c \in X^{\prime}$, so that $\mathrm{cf}(\kappa) \leq \gamma$ in $K(\mathcal{F})$, contrary to assumption.

Now if $\alpha=\inf \left(\left\{\alpha^{N}(c): c \in E\right\}\right)$ then $\alpha^{N}(c)=\alpha$ for all sufficiently large $c \in E$, so we may as well assume $\alpha^{N}(c)=\alpha$ for all $c \in E$.

Suppose that $\bigcup_{c \in E} \beta^{N}(c)<\delta$. Then there is a fixed $\beta<\delta$ and a stationary subset $X$ of $E$ of ordinals of cofinality $\omega$ such that $\beta^{N}(c)=\beta$ for $c \in X$. Let $c$ be any limit point of $X$ in $X$. Since $N$ contains all of its countable subsets, Lemma 1.6 implies that $\beta^{N}(c)>\beta$; this contradiction shows that $\beta^{N}(\alpha) \geq \delta$.

We want to show that $o(\kappa)$ itself is at least $\delta$. If we had assumed that $2^{\delta}<\kappa$ then we could assume ${ }^{\delta} N \subset N$ and apply Lemma 1.6 directly, but as it is we do not know that $E \in N$. By using Lemma 1.6 together with the fact that ${ }^{\omega} N \subset N$ we can find an $\omega$-closed, unbounded sequence $\left(c_{\nu}: \nu<\delta\right)$ so that $\alpha^{N}\left(c_{\nu}\right)=\alpha$ and $\beta^{N}\left(c_{\nu^{\prime}}\right)<$ $\beta^{N}\left(c_{\nu}\right)$ when $\nu^{\prime}<\nu<\delta$. Pick $N^{\prime} \supset N$ such that $\left(c_{\nu}: \nu<\delta\right)$ and $\left(\beta_{\nu}: \nu<\delta\right)$ are in $N^{\prime}$. By Lemma 1.4 we have $c_{\nu} \in C^{N^{\prime}}\left(c_{\nu+1}, C\left(\alpha, \beta^{N}\left(c_{\nu}\right), \beta^{N}\left(c_{\nu+1}\right)\right)\left(c_{\nu+1}\right)\right)$ for all sufficiently large $\nu$. It follows that $\alpha^{N^{\prime}}\left(c_{\nu}\right)=\alpha$ for some fixed $\alpha$ and all sufficiently large $\nu$. If $\alpha$ has repeat points then it is not clear that $\left(\beta^{N^{\prime}}\left(c_{\nu}\right): \nu<\delta\right) \in N^{\prime}$ so we still cannot apply Lemma 1.6 directly, but since $\sup \left(o\left(c_{\nu}\right): \nu<\delta\right) \geq \delta$ we can apply the proof of Lemma 1.6 to see that $o(\kappa) \geq \delta$.

This completes the proof of the general statement of Theorem 0.1. The main statement follows immediately from this: if there is no inner model of $\exists \kappa o(\kappa) \geq \delta$ then $K(\mathcal{F})$ exists, and by Lemma 7.14 of $[\mathbf{M i}], \mathcal{F}$ is still maximal and $K(\mathcal{F})$ is still its core model in any generic extension $V(G)$ of $V$. The general statement, applied in $V(G)$, then implies that the hypothesis of Theorem 0.1 implies $o(\kappa) \geq \delta$ in $K(\mp)$.

PROOF OF THEOREM 0.2 . Let $\kappa$ be the true $\omega_{1}$. We are given that the filter $C$ of closed, unbounded subsets of $\kappa$ is an ultrafilter. By the covering lemma, $C \cap K(\mathcal{F})=\mathcal{F}(\kappa, \beta)$ for some $\beta<o(\kappa)$; we will show that $\beta$ is a repeat point. Suppose that it is not. Then there is a set $A$ in $K(\mathcal{F})$ such that $A \in \mathcal{F}(\kappa, \beta)$ but $A \notin \mathcal{F}\left(\kappa, \beta^{\prime}\right)$ for all $\beta^{\prime}<\beta$. We can assume that every ordinal in $A$ is inaccessible in $K(\mathcal{F})$ and that for all $\alpha \in A, A \cap \alpha \notin \bigcup_{\gamma} \mathcal{F}(\alpha, \gamma)$. Since $\mathcal{F}(\kappa, \beta)=C, A$ contains a closed, unbounded set $c$. Let $\mathbf{M}=L^{C}(K(\mathcal{F}), c)$, the smallest model containing 
the set $c$, the class $K(\mathcal{F})$, and $C \cap \mathbf{M}$. For the rest of the proof we will be working inside $\mathbf{M}$. Since $\kappa$ is measurable in $\mathbf{M}$ we can find a cardinal $\alpha$ in $c$ of cofinality $\omega_{1}^{(\mathbf{M})}$. Now $K(\boldsymbol{\xi})$ is still the core model inside $\mathbf{M}$ and $\alpha$ is regular in $K(\mp)$. Pick $N$ so that $|N|<\alpha,{ }^{<\alpha} N \subset N$, and $N^{\prime} \cap \alpha$ is unbounded in $\alpha$. Then $C^{N}$ contains a closed, unbounded set of indiscernibles for measures on $\alpha$, and since $c$ is also closed and unbounded, unboundedly many of them must be in $c$ and hence in $A$. It follows that $A \cap \alpha$ is in some measure on $\alpha$, contradicting the assumption on $A$.

2.1. Problems. (i) In $[\mathbf{M i 8 2}]$ we show that if $o(\kappa)=\beta+1$ where $\beta$ is a limit of $\kappa^{+}$strong repeat points, then there is a generic extension in which the closed unbounded filter is an ultrafilter. What is the true consistency strength of this condition?

(ii) Under the assumption of $\mathrm{AD}$ the ultrafilter $C$ of closed, unbounded sets has the stronger property that $i^{C}\left(\omega_{1}\right)=\omega_{2}$. What is the consistency strength of this property? It is probably stronger than $o(\kappa)=\kappa^{++}$since if we set $\kappa=\omega_{1}$ then it implies that $\kappa^{+}$of $K(₹)$ has cardinality $\kappa$ in the real world, so that all the measures on $\kappa$ in $K(\mp)$ are still in $V^{\kappa} / C$. Only a slight strengthening of the covering lemma would be required to show that this is impossible.

3. Introduction to $L^{j}$. This section is preliminary to the more difficult material in the last two sections. We will end the section with the proof of Theorem 0.3 but first we will consider, as an aid to the intuition, a simple case of Theorem 0.4. Suppose that $U$ and $W$ are measures on $\kappa$ and $\lambda$, respectively, that $V=L(U, W)$, and $j=i^{U}: V \rightarrow V^{\kappa} / U$.

3.1. Proposition. (i) $L^{j}$ is obtained by taking $\kappa$ iterations of the measure $W:$

$$
i=i_{\kappa}^{W}: L(U, W) \rightarrow L^{j}=\operatorname{ult}_{\kappa}(L(U, W), W) .
$$

(ii) The embedding $j \uparrow L^{j}$ is obtained inside $L^{j}$ by taking a single ultrapower of $L^{j}$ by $i(U)$, followed by $j(\kappa)$ iterations by the measure $i(W)$.

ProOF. We first observe that $i(U)$ and $i(W)$ are in $L^{j}$. The second is easy: if $\vec{\lambda}=\left(\lambda_{\nu}: \nu \in \kappa\right)$ are the iteration points of $i, \lambda_{\nu}=i_{\nu}^{W}(\lambda)$, then $x \in i(W)$ iff $j(\vec{\lambda})_{\kappa} \in j(\kappa)$. There are two slightly different cases for $i(U)$, depending on whether $\kappa<\lambda$ or $\kappa>\lambda$. If $\kappa<\lambda$ then $i(\kappa)=\kappa$ and $i(U)=U=\{x: \kappa \in j(x)\}$. If $\kappa>\lambda$ then $i(\kappa)>\kappa$ but since each $x \in P(i(\kappa)) \cap$ ult $_{\kappa}(L(U, W), W)$ has a support bounded in $\kappa$ we get $x \in i(U)$ iff $j(i \uparrow \kappa)(\kappa) \in j(x)$, so again $i(U) \in L^{j}$.

In this later case we actually have $U \in L^{j}$, just as in the first case, but this is not helpful: in fact $U \cap L^{j}=i(W)$.

It follows that $M=L(i(U), i(W)) \subset L^{j}$. Clause (ii) of the lemma implies that $j \uparrow M \in M$, so that $L^{j} \subset M$ and hence $L^{j}=M$. Thus the proof of clause (ii) is the crucial step which will also complete the proof of clause (i) and hence of the theorem.

Set $j^{*}=i_{j(k)}^{i(W)} i^{i(U)}$, so that clause (ii) asserts that $j \uparrow M=j^{*}$. If $x$ is any member of $M$ then $x$ can be written in the form $i(f)(\vec{c})$ where $\vec{c}$ is a sequence of iteration points $i_{\nu_{i}}^{W}(\lambda)$ of $\lambda$. It is easy to verify that $j(\vec{c})=\vec{c}=j^{*}(\vec{c})$ (this verification is most easily carried out separately for the cases $\kappa<\lambda$ and $\lambda<\kappa)$. Thus $j(x)=j(i(f)(\vec{c}))=j(i(f))\left(j^{*}(\vec{c})\right)$ and if we show that $j i=j^{*} i$ then we will 
have

$$
j(x)=j i(f)\left(i^{*}(\vec{c})\right)=j^{*} i(f)\left(j^{*}(\vec{c})\right)=j^{*}(i(f)(\vec{c}))=j^{*}(x),
$$

as required. Now $j i=j(i) j=j\left(i_{\kappa}^{W}\right) j=i_{j(\kappa)}^{j(W)} j$; that is to say, a single ultrapower by $U$ followd by $j(\kappa)$ ultrapowers using $W$. On the other hand $j^{*} i$ involves $\kappa$ iterations using $W$, then a single iteration by $U$, and then $j(\kappa)$ more iterations using $W$. Since ultrapowers on different cardinals commute we can move the ultrapower by $U$ to the front, so this is the same as a single interation by $U$ followed by $j(\kappa)$ iterations by $W$, which is the same as $j i$.

The full proof of Theorem 0.4 is, of course, more complicated because it must handle more complicated sequences, but a second major difficulty enters as soon as there are cardinals $\lambda$ such that $o(\lambda) \geq \kappa$ in $K(\mathcal{F})$. The argument above used the fact that $j(W)=W$. As long as $\operatorname{cf}(\lambda) \neq \kappa$ for all $\lambda$ (except $\kappa$ ) which are measurable in $K(\mathcal{F})$ then we can use a reasonably straightforward generalization of the argument of Proposition 3.1; in particular $L^{j}$ is obtained in the same way, by taking $\kappa$ iterations using each measure in $K(\mathcal{F})$ except those on $\kappa$. If $\operatorname{cf}(\lambda)=$ $\kappa$, which can happen when $o(\lambda) \geq \kappa$, the measures on $\lambda$ may already be in $L^{j}$, without having to take the $\kappa$ iterations. The need to distinguish these possibilities is responsible for much of the difficulty in the proof of Theorem 0.4.

PROOF OF THEOREM 0.3. Let $K^{j}\left(\mathcal{F}^{j}\right)$ be the maximal core model as defined in $L^{j}$. We will first show that every $\mathcal{F}^{j}$-mouse is in $L^{j}$, so that $K^{j}\left(\mathcal{F}^{j}\right)=K\left(\mathcal{F}^{j}\right)$, and then we will show that $K\left(\mathcal{F}^{j}\right)$ is an iterated ultrapower of $K(\mathcal{F})$.

Suppose that there is an $\mathcal{F}^{j} \uparrow \gamma$-mouse $m=J_{\eta}^{\mathcal{G}}$ which is not in $L^{j}$, and assume that $\gamma$ is minimal. We define iterated ultrapowers $i: K^{j}\left(\mathcal{F}^{j}\right) \rightarrow K_{\nu}\left(\mathcal{F}_{\nu}\right)$ and $k: m \rightarrow J_{\eta^{\prime}}^{k(\mathcal{G})}$ as usual: $i$ and $k$ are limits of the sequence $i_{\nu}: K^{j}\left(\mathcal{F}^{j}\right) \rightarrow K\left(\mathcal{F}_{\nu}\right)$ and $k_{\nu}: J_{\eta}^{\mathcal{G}} \rightarrow m_{\nu}=J_{\eta_{\nu}}^{\mathcal{G}_{\nu}}$, where $i_{\nu, \nu+1}$ and $k_{\nu, \nu+1}$ are either the identity or the ultrapower by $\mathcal{F}_{\nu}\left(\alpha_{\nu}, \beta_{\nu}\right)$ and $\mathcal{G}_{\nu}\left(\alpha_{\nu}, \beta_{\nu}\right)$, respectively, and $\left(\alpha_{\nu}, \beta_{\nu}\right)$ is the least pair such that either $\mathcal{F}_{\nu}\left(\alpha_{\nu}, \beta_{\nu}\right) \neq \mathcal{G}_{\nu}\left(\alpha_{\nu}, \beta_{\nu}\right)$ or $\beta_{\nu}=\inf \left(o^{\mathcal{G}_{\nu}}\left(\alpha_{\nu}\right), o^{\mathcal{F}_{\nu}}\left(\alpha_{\nu}\right)\right)<$ $\sup \left(o^{\mathcal{G}_{\nu}}\left(\alpha_{\nu}\right), o^{\mathcal{F}_{\nu}}\left(\alpha_{\nu}\right)\right)$. If $k_{\nu, \nu+1}$ is the identity for all sufficiently large $\nu$ then $m_{\nu}$ is an initial segment of $L\left(\mathcal{F}_{\nu}\right)$, which implies that $m_{\nu} \in L\left(\xi_{\nu}\right)$ and hence (since $m$ is isomorphic to the transitive collapse of a definable submodel of $m_{\nu}$ ) implies that $m$ is in $K^{j}\left(\mathcal{F}^{j}\right)$. Thus the comparison never terminates.

This comparison is slightly different from those of [Mi84a, $\mathbf{M i}$ ], since $₹$ may be a proper class. Start with the class $D$ of ordinals of cofinality $\kappa$. We will put a series of additional conditions on the ordinals in $D$ which will shrink it to a smaller, but still stationary, class. First, since each $m_{\nu}$ is a set we can assume that there are ordinals $\nu_{0}$ and $\alpha, \beta \in m_{\nu_{0}}$ so that $\alpha_{\nu}=k_{\nu_{0}, \nu}(\alpha)$ and $\beta_{\nu}=k_{\nu_{0}, \nu}(\beta)$ for each $\nu \in D$. Then $k_{\nu^{\prime}, \nu}\left(\alpha_{\nu^{\prime}}, \beta_{\nu^{\prime}}\right)=\left(\alpha_{\nu}, \beta_{\nu}\right)$ for $\nu^{\prime}<\nu$ in $D$. It follows that $k_{\nu, \nu+1}\left(\alpha_{\nu}\right)>\alpha_{\nu}$ for each $\nu \in D$, so $o^{\mathcal{G}_{\nu}}\left(\alpha_{\nu}\right)>\beta_{\nu}$. It also follows that we can shrink $D$ to a stationary subclass so that we have $i_{\nu}(\nu)=\nu=\alpha_{\nu}$ for all $\nu \in D$.

We have two cases. If $o^{\mathcal{F}_{\nu}}\left(\alpha_{\nu}\right)>\beta_{\nu}$ for a stationary class, then there is a set $x_{\nu} \in m_{\nu} \cap K\left(\mathcal{F}_{\nu}\right)$ such that $x_{\nu} \in \mathcal{G}_{\nu}\left(\alpha_{\nu}, \beta_{\nu}\right) \backslash \mathcal{F}_{\nu}\left(\alpha_{\nu}, \beta_{\nu}\right)$ for each $\nu$, and we can shrink $D$ to a stationary subclass such that $x_{\nu}=k_{\nu_{0}, \nu}(x)$ for all $\nu \in D$ and some fixed $\nu_{0}$ and $x$. Then $k_{\nu^{\prime}, \nu}\left(x_{\nu^{\prime}}\right)=k_{\nu^{\prime}, \nu}\left(k_{\nu_{0}, \nu^{\prime}}(x)\right)=k_{\nu_{0}, \nu}(x)=x_{\nu}$. Because $x_{\nu^{\prime}} \in \mathcal{G}_{\nu}\left(\alpha_{\nu}, \beta_{\nu}\right)$ we then have $\nu^{\prime} \in k_{\nu^{\prime}, \nu}\left(x_{\nu^{\prime}}\right)=x_{\nu}$; and also we have $x_{\nu^{\prime}}=x_{\nu} \cap \nu^{\prime}$. Now shrink $D$ again so that for all $\nu \in D$ and some fixed $\nu_{0}$ there is $\bar{x}_{\nu} \in K_{\nu_{0}}\left(\mathcal{F}_{\nu_{0}}\right)$ 
such that $x_{\nu}=i_{\nu_{0}, \nu}\left(\bar{x}_{\nu}\right)$. Then

$$
i_{\nu_{0}, \nu}\left(\bar{x}_{\nu}\right) \cap \nu^{\prime}=x_{\nu} \cap \nu^{\prime}=x_{\nu^{\prime}}=i_{\nu_{0}, \nu}\left(\bar{x}_{\nu^{\prime}}\right) \cap \nu^{\prime}
$$

so $\bar{x}_{\nu^{\prime}}=\bar{x}_{\nu} \cap \nu^{\prime}$. But then $x_{\nu^{\prime}} \notin \mathcal{F}_{\nu}\left(\alpha_{\nu}, \beta_{\nu}\right)$ implies that

$$
\nu^{\prime} \notin i_{\nu^{\prime}, \nu}\left(x_{\nu^{\prime}}\right)=i_{\nu_{0}, \nu}\left(\bar{x}_{\nu^{\prime}}\right)=i_{\nu_{0}, \nu}\left(\bar{x}_{\nu} \cap \nu^{\prime}\right)=x_{\nu} \cap i_{\nu^{\prime}, \nu}\left(\nu^{\prime}\right),
$$

contradicting the fact that $\nu^{\prime} \in x_{\nu}$. This contradiction proves that we can shrink $D$ to a stationary subclass so that for all $\nu \in D$ we have $i(\nu)=\nu$ and hence $o^{\mathcal{F}_{\nu}}\left(\alpha_{\nu}\right)=\beta_{\nu}$.

Let $\nu$ be a limit point of $D$ in $D$. Then $U=\mathcal{G}_{\nu}\left(\alpha_{\nu}, \beta_{\nu}\right)=\{x \subset \lambda: D \backslash x$ is bounded in $\left.\alpha_{\nu}\right\}$, and $U$ is a measure on $K_{\nu}\left(\xi_{\nu}\right)$ since $P\left(\alpha_{\nu}\right) \cap K_{\nu}\left(\xi_{\nu}\right) \subset m_{\nu}$. Since $i_{\nu}\left(\nu^{\prime}\right)=\nu^{\prime}$ for all $\nu^{\prime} \in D, U$ is also a measure on $K^{j}\left(\mathcal{F}^{j}\right)$. Now let $f$ enumerate a cofinal subset of $D \cap \alpha_{\nu}$ of order type $\kappa$. Then any set $x$ is in $U$ iff $[f]_{U} \in j(x)$ so $U$ is in $L^{j}$, contradicting the maximality of $K^{j}\left(\mathcal{F}^{j}\right)$ in $L^{j}$.

Now since $K^{j}\left(\mathcal{F}^{j}\right)=K\left(\mathcal{F}^{j}\right)$, we know by Theorem 7.10 of $[\mathbf{M i}]$ that there is an iterated ultrapower $k$ of $K(\mathcal{F})$ which takes $\mathcal{F}$ (or an initial part of $\mathcal{F}$ ) to $\mathcal{F}^{j}$. We need to show, in the terminology of that paper, that $k$ is proper. If $k$ is the limit of the iterated ultrapowers $k_{\nu}: K(\mathcal{F}) \rightarrow K\left(\mathcal{F}_{\nu}\right)$ then this means that $\left\{k_{\nu}(\alpha): \nu \in\right.$ ord $\}$ is bounded for each ordinal $\alpha$. If $k$ is not proper then there are $\alpha$ and $\nu_{0}$ such that $\left\{k_{\nu_{0}, \nu}(\alpha): \nu \in\right.$ ord $\}$ is unbounded but $\left\{k_{\nu^{\prime}}\left(\alpha^{\prime}\right): \nu \in\right.$ ord $\}$ is bounded for each $\nu^{\prime}>\nu_{0}$ and $\alpha^{\prime}<k_{\nu_{0}, \nu}(\alpha)$. Then $\alpha_{\nu}=k_{\nu_{0}, \nu}(\alpha)$ for a closed and unbounded class $D$, and we can shrink $D$ to a stationary subclass of ordinals of cofinality $\kappa$ such that $k_{\nu^{\prime}, \nu}\left(\beta_{\nu^{\prime}}\right)=\beta_{\nu}$ for $\nu^{\prime}<\nu$ in $D$. Then the argument of the second case above shows that if $\nu$ is a limit point of $D$ in $D$ then $\xi_{\nu}\left(\alpha_{\nu}, \beta_{\nu}\right) \in L^{j}$, contradicting the maximality of $\mathcal{F}^{j}$ there.

4. The proof of Theorem 0.4. In this section we introduce the main concepts of the proof and complete the proof except for the main lemma. The main lemma, which is stated near the end of this section and proved in the next section, will require a large part of our effort.

We have $U$ a measure on $\kappa$ and $j: V \rightarrow V^{\kappa} / U$. By Lemma 7.16 of $[\mathbf{M i}], j \uparrow K(\mathcal{F})$ is an iterated ultrapower of $K(\mathcal{F})$. Since $\kappa$ is the critical point of $j$, this iterated ultrapower will use no measures on cardinals below $\kappa$, but at least one measure on $\kappa$. Our arguments will be easier if $j$ uses all (or at least cofinally many) of the measures on $\kappa$, therefore we let $\beta^{j} \leq o(\kappa)$ be the least ordinal (if there is any) such that if the iterated ultrapower $j$ is the limit of iterated ultrapowers $j_{\nu}: K(\mathcal{F}) \rightarrow K\left(\mathcal{F}_{\nu}\right)$ then at no stage is an ultrapower by $\mathcal{F}_{\nu}\left(j_{\nu}(\kappa), \beta^{\prime}\right)$ used for $\beta^{\prime} \geq j_{\nu}\left(\beta^{j}\right)$. Equivalently, $\beta^{j}$ is the least ordinal such that $C^{j}\left(j(\kappa), \beta^{\prime}\right)=\varnothing$ for all $\beta^{\prime}>\beta^{j}$ where $C^{j}$ is the system of indiscernibles generated by $j$. Set $K\left(\mathcal{F}_{j}\right)=\operatorname{ult}\left(K(\mathcal{F}), \mathcal{F}\left(\kappa, \beta^{j}\right)\right)$ if $\beta^{j}<o\left(\beta^{j}\right)$ and $\mathcal{F}_{j}=\mathcal{F}$ otherwise. Then by Lemma 7.8 of $[\mathbf{M i}], K\left(\mathcal{F}_{j}\right)$ is still maximal everywhere except at $\kappa$. In particular $j \uparrow K\left(\mathcal{F}_{j}\right)$ is still an iterated ultrapower of $K\left(\mathcal{F}_{j}\right)$. For the rest of the paper we will be using $K\left(\mathcal{F}_{j}\right)$ exclusively rather than $K(\mathcal{F})$, so we will drop the subscript, using the letter $\mathcal{F}$ to denote what we have been calling $\mathcal{F}_{j}$.

We now must construct some machinery in order to proceed.

4.1. Definition. (i) If $\mathcal{C}=C^{i}$ for some embedding $i: K(\mathcal{F}) \rightarrow K(i(\mathcal{F}))$ then we say that $\beta$ is definable for $C$ from $x$ if there is $f \in K(\mathcal{F})$ such that $\beta \in i(f)^{\prime \prime} x$. If $C=C^{N}$ then $\beta$ is definable for $C$ from $x$ if $\beta \in h^{N \prime \prime} x$. 
(ii) Suppose that $C$ is a system of indiscernibles for the sequence $\mathcal{G}$. Then we say that $c$ is an accumulation point in $C$ for measures on $\alpha$ below $\beta$ if $\beta$ is definable for $C$ from $c$ and for all $\nu<c$ and $\gamma<\beta$ such that $\gamma$ is definable for $C$ from $c$ there is $c^{\prime} \in C\left(\alpha, \beta^{\prime}\right)$ with $\nu<c^{\prime} \leq c$ and $\gamma \leq \beta^{\prime}<\beta$. (Notice that this definition includes the case when $\beta=\beta^{\prime}+1$ and $c \in C\left(\alpha, \beta^{\prime}\right)$.)

(iii) $D$ is a $\delta$-set of indiscernibles for the measure $\mathcal{G}(\alpha, \beta)$ if $D$ has order type and $\mathcal{G}(\alpha, \beta)=\{x: D \backslash x$ is bounded in $\alpha\}$.

(iv) More generally, if $\vec{c}=\left(c_{\nu}: \nu<\delta\right)$ is an increasing sequence of ordinals below $\alpha$ and $\vec{\beta}=\left(\beta_{\nu}: \nu<\delta\right)$ is a nondecreasing sequence of ordinals below $o(\alpha)$ then we say that $\vec{c}$ is a $\delta$-set of indiscernibles for the measures $\vec{\beta}$ on $\alpha$ (or, more loosely, on the measures below $\beta=\bigcup \vec{\beta}$ ) provided that $\vec{c}$ is unbounded in $\alpha$, there is a function $g \in K(\mathcal{F})$ such that each $\beta_{\nu} \in g^{\prime \prime} c_{\nu}$, and $\left\{\nu \in \delta: \exists x \in f^{\prime \prime} c_{\nu} c_{\nu} \in x\right.$ $\left.x \in \mathcal{G}\left(\alpha, \beta_{\nu}\right)\right\}$ is bounded in $\delta$ for each function $f \in K(\mathcal{F})$.

We will say that there is a $\delta$-set of indiscernibles for cofinally many measures below $\beta$ if there are $\delta$-sets $\vec{c}$ for sequences $\vec{\beta}$ with $\sup (\vec{\beta})$ arbitrarily large in $\beta$. Notice that if $C$ is a complete set of indiscernibles for the measures below $\alpha$ then this implies that there are $\delta$-sets of indiscernibles for every measure below $\beta$.

4.2. PROPOSITION. If $c$ is an accumulation point in $C^{j}$ for measures on $\alpha$ below $\beta$ and $\operatorname{cf}(c) \leq \kappa$ then $c \in C^{j}\left(\alpha, \beta^{\prime}\right)$ for some $\beta^{\prime} \geq \beta$.

PROOF. For each $\gamma<\beta$ which is definable for $C^{j}$ from $c$ let $x \subset c$ be in $U_{\gamma}$ iff the set of $\nu<c$ such that

$$
\exists \lambda>\gamma\left(\nu \in C^{j}(\alpha, \lambda) \text { and } x \cap \nu \in j(\mathcal{F})(\nu, C(\alpha, \gamma, \lambda)(\nu))\right)
$$

is unbounded in $c$. Then each $U_{\gamma}$ is a measure on $c \cap K(j(\mathcal{F}))$. It is in $V^{\kappa} / U$ because it can be defined from a sequence of length of at most $\kappa$ and $V^{\kappa} / U$ is closed under sequences of length $\kappa$. It follows by maximality that it is in the sequence $j(\mathcal{F})$. Then $U_{\gamma}=j(\mathcal{F})(c, C(\alpha, \gamma, \beta)(c))$ and so $c \in C^{j}\left(\alpha, \beta^{\prime}\right)$ for some $\beta^{\prime}>\gamma$.

\subsection{COROLlARY. $o^{\mathcal{F}}(\kappa)$ is either a successor or of cofinality at least $\kappa$.}

PROOF. If $\operatorname{cf}\left(o^{\mathcal{F}}(\kappa)\right)<\kappa$ in $K(\mathcal{F})$ then $j^{\prime \prime} o(\kappa)$ is cofinal in $j(o(\kappa))$. By the choice of $\mathcal{F}$, there must be an accumulation point in $C^{j}$ for measures on $j(\kappa)$ below $j(o(\kappa))$. The least such accumulation point has cofinality $\operatorname{cf}\left(o^{\mathcal{F}}(\kappa)\right)$ and hence cannot be less than $j(\kappa)$ by Proposition 4.2 , but it cannot be $j(\kappa)$ because $j(\kappa)$ has cofinality greater than $\kappa$.

We write $N\left(\alpha, \beta, \delta, \nu, \beta^{\prime}\right)$ to mean the least member of $C\left(\alpha, \beta^{\prime}\right)$ which is greater than or equal to the $\nu$ th ordinal $c>\delta$ such that $c$ is an accumulation point in $C$ for measures on $\alpha$ below $\beta$. Notice that the next indiscernible function, $s(\alpha, \beta, \delta)=$ $\bigcap(C(\alpha, \beta) \backslash \delta+1)$, may be written $N(\alpha, \beta+1, \delta, 1, \beta)$.

4.4. PROPOSITION. Every indiscernible $c$ in $C^{j}$ may be written in the form $N(\alpha, \beta, \delta, 1, \lambda)$ for some $\delta<c$, and some $\alpha, \beta$, and $\lambda<\beta$ which are definable for $c^{j}$ from $c$.

Proof. By the definition of accumulation point, there is a largest ordinal $\beta$ so that $c$ is an accumulation point for measures below $\beta$. If $\beta=\lambda+1$ and $c=$ $s(\alpha, \lambda, \beta)=N(\alpha, \beta, \delta, 1, \lambda)$ then we are done. Otherwise the fact that $c \notin C^{j}\left(\alpha, \beta^{\prime}\right)$ 
for any $\beta^{\prime} \geq \beta$ implies that $\operatorname{cf}(c)>\omega$ by Proposition 4.2. If $c \neq N(\alpha, \beta, \delta, 1, \lambda)$ for any $\delta<c$ and $\lambda<\beta$ then the set $A$ of accumulation points for measures below $\beta$ must be closed and unbounded in $c$. By Proposition 4.2 every limit point of $A$ of cofinality $\omega$ is in $C^{j}\left(\alpha, \beta^{\prime}\right)$ for some $\beta^{\prime} \geq \beta$. Then $c$ is an accumulation point $\beta^{\prime}+1$, contradicting the choice of $\beta$.

The parameter $\nu$ was not required here because of the fact that there are never more than $\omega$ consecutive accumulation points for the same measure without accumulation points for larger measures. It will be required later for the iterated ultrapower $i^{*}$, which will have $\kappa$ consecutive accumulation points. The parameter $\lambda$ was not really required here either, since $c$ was always the least accumulation point for $\beta$ and $\lambda$ was simply taken to be the ordinal such that $c \in C(\alpha, \lambda)$. To see why we nevertheless need to include $\lambda$, consider an iterated ultrapower $k: K(j(\mathcal{F})) \rightarrow M$ which moves $c$. Then $k(c)$ will no longer be an accumulation point for $k(\beta)$ in $C^{k j}$, but $k(c)$ will be $N^{k j}(k(\alpha), k(\beta), k(\delta), 1, k(\lambda))$.

4.5. Definition. (i) A support in $C^{j}$ is an increasing sequence $\vec{c}=\left(c_{0}, \ldots, c_{n-1}\right)$ such that each $c_{m}$ is equal to $N\left(\alpha_{m}, \beta_{m}, c_{m-1}, 1, \beta_{m-1}^{\prime}\right)$ for some $\alpha_{m}, \beta_{m}$, and $\beta_{m}^{\prime}$ definable for $c^{j}$ from $c \nmid m$, with $\beta_{m}$ as large as possible. (If $m=0$, then we take $c_{m-1}$ to be 0 .) The support functions for $\vec{c}$ are the functions witnessing that the ordinals $\alpha_{m}, \beta_{m}$, and $\beta_{m}^{\prime}$ are definable.

(ii) A support for a set $x \in K(j(\mathcal{F}))$ is a support $\vec{c}$ in $C^{j}$ such that $x$ is definable for $C^{j}$ from $\vec{c}$. The support functions for $x$ are the support functions of $\vec{c}$ together with the function witnessing the definability of $x$ from $\vec{c}$.

We will use the accumulation points of $j$ to find a finite piece of information which characterizes the action of $j$ at $\kappa$. Once we have this characterization we will be ready to give the construction of $L^{j}$ and $j \uparrow L^{j}$.

4.6. DEFInition. The increasing sequence $\left(c_{n}: n<k\right)$ of ordinals below $j(\kappa)$ and nonincreasing sequence $\left(\beta_{n}: n<k\right)$ of measures on $j(\kappa)$ are defined by induction on $n: \beta_{n}$ is the largest ordinal such that there is an accumulation point $c$ in $C^{j}$ for measures below $\beta_{n}$. with $c_{n-1}<c \leq j(\kappa)$, and $c_{n}$ is the smallest such accumulation point.

We claim that $k$ is finite. Otherwise, since $\left(\beta_{n}: n<k\right)$ is nonincreasing it must be eventually constant. Let $\beta=\bigcap_{n} \beta_{n}$, the eventual value of $\beta_{n}$, and $c=\bigcup_{n} c_{n}$. Then $c<j(\kappa)$ since $j(\kappa)$ has cofinality greater than $\kappa$. Now $c$ is an accumulation point for measures below $\beta$ so by Proposition $4.2, c \in C^{j}\left(\alpha, \beta^{\prime}\right)$ for some $\beta^{\prime} \geq \beta$ and hence is an accumulation point for measures below $\beta^{\prime}+1$, contradicting the choice of $c_{n}$.

These sequences do not, of course, actually determine $j$, either at $\kappa$ or elsewhere. They will, however (or rather the support functions for $\vec{c}$ will), determine $j \uparrow L^{j}$. In fact $L^{j}$ will depend only on $K(\mathcal{F})$ and hence on the ordinal $\beta^{j}$ defined earlier, and $j \uparrow L^{j}$ will depend only on $L^{j}$ and the support functions for the sequence $\left(c_{n}: n<k\right)$. Thus the class $L^{j}$ will be the same for all of the four examples below, and $j \uparrow L^{j}$ will be the same for all but the last. We assume that $o^{\mathcal{F}}(\kappa)=2$ and let $U_{0}=\mathcal{F}(\kappa, 0)$ and $U_{1}=\mathcal{F}(\kappa, 1)$.

(1) The model $L(\mathcal{F})$, with $j$ being the ultrapower by $U_{1}=\mathcal{F}(\kappa, 1)$.

(2) Let $j$ be the iterated ultrapower by $U_{0} \times U_{1}$ and use methods of Kunen and Paris [K-P71] to get a generic extension of $L(\mathcal{F})$ in which $j$ is the ultrapower by an extensior of $U_{1}$. 
(3) Let $j$ be the iterated ultrapower using $\omega$ many interations of $U_{0}$ and a single interation of $U_{1}$. Obtain a model in which $j$ is the canonical embedding of an extension of $U_{1}$ by first using iterated Prikry forcing [Ma78] to make every measurable cardinal below $\kappa$ have cofinality $\omega$ and then using the Kunen-Paris technique.

(4) Let $j$ be the iterated ultrapower by $U_{1}^{2}$ and proceed as in example (2).

Our basic result is

4.7. THEOREM. Suppose that there is no inner model of $\exists \kappa o(\kappa)=\kappa^{++}$, that $U$ is a measure on $\kappa$, and that $j: V \rightarrow V^{\kappa} / U$ is the canonical embedding. Then $L^{j}=K\left(\mathcal{F}^{*}\right)$ and $j \uparrow L^{j}=j^{*}$, where $\mathcal{F}^{*}$ and $j^{*}$ are defined as follows:

4.8. Definition. $i^{*}: K(\mathcal{F}) \rightarrow K\left(\mathcal{F}^{*}\right)$ is the least iterated ultrapower such that every measure in $\mathcal{F}^{*}$ except those on $i^{*}(\kappa)$ has a $\kappa$-set of indiscernibles.

Notice that the $\kappa$-set of indiscernibles may either be in $V$ to start with or it may be added by $i^{*}$. In the simplest cases, when $V=L(\mathcal{F})$ or $o(\lambda)<\kappa$ in $K(\mathcal{F})$ for all cardinals $\lambda$, there are no $\kappa$-sets of indiscernibles in $V$ and so they must all be added by $i^{*}$.

4.9. Definition. The map $j^{*}$ is defined in $K\left(\mathcal{F}^{*}\right)$ in two parts,

$$
j^{*}: K\left(\mathcal{F}^{*}\right) \stackrel{j_{1}}{\rightarrow} K\left(j_{1}\left(\mathcal{F}^{*}\right)\right) \stackrel{j_{2}}{\rightarrow} K\left(\mathcal{F}^{* *}\right) .
$$

The first, $j_{1}$, incorporates the effect of $j$ at $\kappa$, while $j_{2}$ is an analogue of $i^{*}$. The second is simpler and we describe it first: simply iterate the measures in $K\left(j_{1}\left(\mathcal{F}^{*}\right)\right)$ until all the measures, except those on $j^{*} i^{*}(\kappa)$, have a $j(\kappa)$-set of indiscernibles. Notice that, unlike the case of $i^{*}$ where some of the sets of indiscernibles may already be in the ground model $V$, the definition of $j_{2}$ takes place inside of $K\left(\mathcal{F}^{*}\right)$ which has no sets of indiscernibles so that all the sets of indiscernibles will be added by the iterated ultrapower $j_{2}$.

The map $j_{1}$ is an iterated ultrapower of $K\left(\mathcal{F}^{*}\right)$ with support functions $i^{*}(\vec{f})$ where $\vec{f}$ is the sequence of support functions of the sequence $\left(c_{n}: n<k\right)$ from Definition 4.6. This statement does not uniquely define $j_{1}$, since an accumulation point for ordinals below a limit ordinal $\beta$ may be obtained by taking iterated ultrapowers using any strictly increasing sequence of measures cofinal in $\beta$, however the choice of this iterated ultrapower does not matter (provided it is in $K\left(\mathcal{F}^{*}\right)$ ) because any differences will be absorbed by $j_{2}$, giving rise to the same map $j^{*}$.

4.10. Lemma. If $j \uparrow K\left(\mathcal{F}^{*}\right)=j^{*}$ then $K\left(\mathcal{F}^{*}\right)=K\left(\mathcal{F}^{j}\right)=L^{j}$. Thus it will be enough to prove that $j \uparrow K\left(\mathcal{F}^{*}\right)=j^{*}$.

PROOF. Since $j^{*}$ is definable inside $K\left(\mathcal{F}^{*}\right)$, the assumption implies that $L^{j} \subset$ $K\left(\mathcal{F}^{*}\right)$. Hence by Theorem 0.3 it will be enough to show that every measure $W$ in $\mathcal{F}^{*}$ is in $L^{j}$. If $W$ is a measure on $\lambda \neq i^{*}(\kappa)$ then this is easy: there is a $\kappa$-set $D$ of indiscernibles for $W$, so $W \cap K\left(\mathcal{F}^{*}\right)=\{x \subset \lambda: d \in j(x)\}$ where $d$ is the $\kappa$ th member of $j(D)$.

Since $o(\kappa)$ either is a successor or has cofinality at least $\kappa$ by Corollary 4.3 and $i^{*}$ does not use any measures on $\kappa, i^{* \prime \prime} o(\kappa)$ is cofinal in $i^{*}(o(\kappa))$ and by coherence it will be enough to show that every measure $\mathcal{F}^{*}\left(i^{*}(\kappa), i^{*}(\beta)\right)$ is in $L^{j}$. By our choice of $\mathcal{F}$, the iterated ultrapower $j$ uses a measure on $\kappa$ larger than $\beta$; this means that there is $\beta^{\prime}>j(\beta)$ such that $C^{j}\left(j(\kappa), \beta^{\prime}\right)$ has a member $d$. Suppose $\beta=j(f)(\vec{c})$ 
where $\vec{c}$ is a support in $C^{j}$ and $f$ is in $K(\mathcal{F})$. If $\vec{c}$ has length $n-1$ then let $F$ be the ultrafilter on $\kappa^{n}$ defined by $F=\left\{x \subset \kappa^{n}:(\vec{c}, d) \in j(x)\right\}$. Then $i^{*}(F) \in L^{j}$ since if $x \in K\left(\mathcal{F}^{*}\right)$ then since the support of $x$ in $i^{*}$ involves only measures below $\kappa$ and $F$ is $\kappa$ complete, $x \in i^{*}(F)$ iff $\left\{\vec{a}: i^{*}(\vec{a}) \in x\right\} \in F$ iff $(\vec{c}-d) \in j\left(\left\{\vec{a}: i^{*}(\vec{a}) \in x\right\}\right)$ iff $j\left(i^{*}\right)(\vec{c} \frown d) \in j(x)$. Since $f(\vec{a})>\beta$ almost everywhere, we have $x \in \mathcal{F}(\kappa, \beta)$ iff

$$
\{(\vec{a}, b): x \cap b \in \mathcal{F}(b, C(\kappa, \beta, f(\vec{a}))(b))\} \in F,
$$

so $x \in i^{*}(\xi(\kappa, \beta))$ iff

$$
\left\{(\vec{a}, b): x \cap b \in \mathcal{F}^{*}\left(b, C\left(i^{*}(\kappa), i^{*}(\beta), i^{*}(f)(\vec{a})\right)(b)\right)\right\} \in i^{*}(F),
$$

and since $\mathcal{F}^{*}\left\lceil i^{*}(\kappa) \in L^{j}\right.$ we have $i^{*}(\mathcal{F}(\kappa, \beta)) \in L^{j}$.

Now if $x \in K\left(\mathcal{F}^{*}\right)$ then $x$ may be written in the form $i^{*}(f)(\vec{c})$, where $f \in K(\mathcal{F})$ and $\vec{c}$ is a finite sequence of indiscernibles from $c^{i^{*}}$. Then $j(x)=j\left(i^{*}(f)(\vec{c})\right)=$ $\left(\left(j\left(i^{*}\right) j\right)(f)\right)(j(\vec{c}))$ and $j^{*}(x)=j^{*}\left(i^{*}(f)(\vec{c})\right)=\left(j^{*} i^{*}(f)\right)\left(j^{*}(\vec{c})\right)$, so the theorem follows immediately from our main lemma:

4.11. LEMMA. (i) $\left(j\left(i^{*}\right) j\right) \uparrow K(\boldsymbol{F})=j^{*} i^{*}$.

(ii) $j(c)=j^{*}(c)$ for any of the indiscernibles $c$ in $c^{i^{*}}$.

The proof of Lemma 4.11 will take up the final section of this paper.

\section{The proof of the main lemma.}

PROOF OF LEMMA 4.11(i). $\left(j\left(i^{*}\right) j\right) \uparrow K\left(\mathcal{F}^{*}\right)=j^{*} i^{*}$.

The two iterated ultrapowers $j\left(i^{*}\right) j$ and $j^{*} i^{*}$ are best understood by looking at the induced systems of indiscernibles. The map $j^{*} i^{*}$ is relatively easy to understand. First, for every measure except those on $\kappa$, the map $i^{*}$ either adds a $\kappa$-set of indiscernibles or does nothing, depending on whether there is already such a set in $V$. In either case $j^{*}$ adds a $j(\kappa)$-set, and the $\kappa$-set from $i^{*}$, if present, will be absorbed into it without changing it. Thus $j^{*} i^{*}$ is, except at $\kappa$, the minimal iteration to generate $j(\kappa)$-sets of indiscernibles for each measure except those on $j^{*} i^{*}(\kappa)$. The map $i^{*}$ adds no indiscernibles to $\kappa$, and $j^{*}$ adds indiscernibles to give $j^{*} i^{*}(\kappa)$ the accumulation points specified by the support functions for the accumulation points in $C^{j}$ at $j(\kappa)$. Because of the completeness of the system of indiscernibles away from $j^{*} i^{*}(\kappa)$ these accumulation points completely characterize the action of $j^{*} i^{*}$ at $\kappa$.

Since $j\left(i^{*}\right)$ does nothing with $j(\kappa), j\left(i^{*}\right) j$ also has the accumulation points specified by the support functions for those for $\kappa$ in $C^{j}$. We will be spending most of the rest of the paper proving that away from $\kappa, j\left(i^{*}\right) j$ is minimal for adding $j(\kappa)$-sets of indiscernibles for every measure except those on $j\left(i^{*}\right) j(\kappa)$, and thus that $j\left(i^{*}\right) j$ is the same as $j^{*} i^{*}$.

Recall that $j\left(i^{*}\right)$ is the iterated ultrapower to add $j(\kappa)$-sets of indiscernibles (except at $\left.j\left(i^{*}\right) j(\kappa)\right)$ whenever they do not exist in $V^{\kappa} / U$. What we want to do is to look at the indiscernibles $C^{j}$ added by $j$ and relate them to the $j(\kappa)$-sets of indiscernibles in $V^{\kappa} / U$. We will show that

(1) Every $j(\kappa)$-set of indiscernibles taken from $C^{j}$ is in $V^{\kappa} / U$. Hence if $j$ generates the required $j(\kappa)$-set of indiscernibles for every measure on $\alpha$ then $j\left(i^{*}\right)$ does nothing. 
(2) Every $j(\kappa)$-set of indiscernibles in $V^{\kappa} / U$ can be taken from $C^{j}$. Hence if $j$ does not generate the required $j(\kappa)$-set of indiscernibles then there is none in $V^{\kappa} / U$ and hence $j\left(i^{*}\right)$ adds one. This set will absorb whatever indiscernibles $j$ did add, so that $j\left(i^{*}\right) j$ adds exactly the required $j(\kappa)$-set of indiscernibles.

We will be applying the techniques of $\S 1$ to the indiscernibles in $C^{j}$ for a cardinal $\alpha \neq j(\kappa)$ of $K(j(\mathcal{F}))$. It turns out that there are two cases, depending on whether $\alpha$ is in the range of $j$.

5.1. LEMMA. Suppose $\alpha=j\left(\alpha^{\prime}\right)$ and $c \in C^{j}(\alpha, \beta)$. If $c=[\lambda \nu c(\nu)]$ and $\beta=[\lambda \nu \beta(\nu)]$ then there is a set $A \in U$ such that $\vec{c}=(c(\nu): \nu \in A)$ is a $\kappa$-set of indiscernibles for $\vec{\beta}=(\beta(\nu): \nu \in A)$ at $\alpha^{\prime}$.

Proof. Pick $g \in K(\mathcal{F})$ so that $\beta \in j(g)^{\prime \prime} c$, let $N \prec H_{\kappa^{++}}$so that $|N|=\kappa$, ${ }^{<\kappa} N \subset N$, and $\kappa+1 \cup\left\{\alpha^{\prime}, \vec{c}, \vec{\beta}\right\} \subset N$, and set $A=$

$$
\left\{\nu: \beta(\nu) \in g^{\prime \prime}(c(\nu)) \text { and } c(\nu) \in C^{N}\left(\alpha^{\prime}, \beta(\nu)\right)\right\} \text {. }
$$

We claim that $A \in U$. If $c(\nu) \in C\left(\alpha^{\prime}, \beta^{\prime}(\nu)\right)$ for some $\beta(\nu) \neq \beta^{\prime}(\nu)$ then let $x(\nu)$ be the least set in $K(\mathcal{F})$ such that $x(\nu) \in \mathcal{F}\left(\alpha^{\prime}, \beta(\nu)\right) \backslash \mathcal{F}\left(\alpha^{\prime}, \beta^{\prime}(\nu)\right)$, and otherwise let $\gamma$ be such that $c(\nu) \leq h^{N}(\gamma)<\alpha^{\prime}$ and let $x(\nu)=\alpha^{\prime} \backslash h^{N}(\gamma)$. Then $x(\nu) \in$ $h^{N \prime \prime} c(\nu) \cap \mathcal{F}\left(\alpha^{\prime}, \beta(\nu)\right)$ for all $\beta \notin A$ such that $\beta(\nu) \in g^{\prime \prime}(c(\nu))$. Thus $x=[\lambda \nu x(\nu)] \in$ $j\left(h^{N}\right)^{\prime \prime} c \cap j(\mathcal{F}(\alpha, \beta))$, while $c \notin x$, contradicting the assumption that $c \in \mathcal{C}^{j}(\alpha, \beta)$. Thus $A \in U$.

We now have to show that $(c(\nu): \nu \in A)$ is a set of indiscernibles. If not then pick $g \in K(\mathcal{F})$ and $\left(x_{\nu}: \nu \in A\right)$ so that $x_{\nu} \in g^{\prime \prime} c_{\nu}$ and $x_{\nu} \in \mathcal{F}\left(\alpha^{\prime}, \beta_{\nu}\right)$ for all $\nu \in A$ but $c_{\nu} \notin x_{\nu}$ for cofinally many $\nu$, and let $N^{\prime} \supset N \cup\{g\} \cup\left\{x_{\nu}: \nu \in A\right\}$. By the argument above, applied in $N^{\prime},\left\{\nu \in A: c_{\nu} \in C^{N^{\prime}}\left(\alpha^{\prime}, \beta_{\nu}\right)\right\}$ is in $U$, and hence is unbounded in $\kappa$, so we can pick out sequences $\left(\nu_{n}: n \in \omega\right)$ and $\left(\xi_{n}: n \in \omega\right)$ such that $\nu>\sup \left(\operatorname{support}(g) \cap \alpha^{\prime}\right)$ and $\nu_{n}<\xi_{n}<\nu_{n+1}, \beta_{\nu_{n}} \leq \beta_{\xi_{n}}, c_{\nu_{n}} \notin x_{\nu_{n}}$, and $c_{\xi_{n}} \in C^{N^{\prime}}\left(\alpha, \beta_{\xi_{n}}\right)$ for each $n \in \omega$. If $\beta_{\nu_{n}}=\beta_{\xi_{n}}$ for infinitely many $n$ then we have $c_{\nu_{n}} \notin x_{\nu_{n}}$ but $c_{\xi_{n}} \in x_{\nu_{n}}$ for infinitely many $n$. By elementarity it follows that there is such a sequence of $x$ 's and function $g$ in $N$, but this is impossible by Fact 1.1(iv)(b). If $\beta_{\nu_{n}}<\beta_{\xi_{n}}$ for infinitely many $n$ then a similar argument leads to a contradiction using Lemma 1.2(iii).

5.2. LEMMA. Suppose that $\vec{c}=\left(c_{\nu}: \nu \in \kappa\right)$ is a $\kappa$-set of indiscernibles for $\vec{\beta}=\left(\beta_{\nu}: \nu \in \kappa\right)$ over $\mathcal{F}$ at $\alpha^{\prime}$ and that $\vec{c}$ and $\vec{\beta}$ are in $N$. Then

(i) $c_{\nu} \in C^{N}\left(\alpha^{\prime}, \beta_{\nu}\right)$ for all sufficiently large $\nu$,

(ii) $j(\vec{c})$ is a $j(\kappa)$-set of indiscernibles for $j(\vec{\beta})$ in $V^{\kappa} / U$, and

(iii) if $\alpha=j\left(\alpha^{\prime}\right)$ and $\kappa \leq \nu<j(\kappa)$ then $j(\vec{c})_{\nu} \in C^{j}(\alpha, j(\vec{\beta})(\nu))$.

PROOF. (i) If $c_{\nu} \notin C^{N}\left(\alpha^{\prime}, \beta_{\nu}\right)$ on an unbounded set then as in the proof of Lemma 5.1 there is a sequence in $N$ of sets $x_{\nu}$ such that $x_{\nu} \in \mathcal{F}\left(\alpha^{\prime}, \beta_{\nu}\right)$ but $c_{\nu} \notin x_{\nu}$ for an unbounded set of $\nu$ 's. This contradicts the assumption that $\vec{c}$ is a $\kappa$-set of indiscernibles for $\vec{\beta}$.

(ii) This is immediate, since $j$ is an elementary embedding.

(iii) Let $f \in \kappa \kappa$ be arbitrary and let $c=\left[\lambda \nu c_{f(\nu)}\right]=j(\vec{c})_{[f]}$. Then $c$ must be in $C^{j}(\alpha, \gamma)$ for some $\gamma<o^{j(\mathcal{F})}(\alpha)$, since otherwise there would be $g \in K(\mathcal{F})$ such that 
$j(g)^{\prime \prime} c \cap[c, \alpha) \neq \varnothing$. It follows that $g^{\prime \prime} c_{\nu} \cap\left[c_{\nu}, \alpha^{\prime}\right) \neq \varnothing$ for cofinally many $\nu<\kappa$ and since $\vec{c} \in N$ there must be such a function $g$ in $N$, which is impossible. Let $\gamma=\left[\begin{array}{ll}\lambda \nu & \gamma_{\nu}\end{array}\right]$ and pick $N^{\prime} \supset N$ as in the proof of Lemma 5.1 so that $\lambda \nu \gamma_{\nu} \in N^{\prime}$. Then the proof of 5.1 shows that $c_{f(\nu)} \in C^{N^{\prime}}\left(\alpha^{\prime}, \gamma_{\nu}\right)$ for almost all $\nu$, but since $\vec{c}$, $\vec{\beta} \in N$ Lemma 1.5(ii) implies that $c_{f(\nu)} \in C^{N^{\prime}}\left(\alpha^{\prime}, \beta_{f(\nu)}\right)$ for all sufficiently large $\nu$. Thus $\gamma_{\nu}=\beta_{f(\nu)}$ for almost every $\nu$ and hence $\gamma=j(\vec{\beta})_{[f]}$.

5.3. LEMMA. Suppose that $\operatorname{cf}\left(o\left(\alpha^{\prime}\right)\right)=\kappa$ and that there are $\kappa$-sets of indiscernibles for a cofinal set of $\beta$ 's in $o\left(\alpha^{\prime}\right)$. Then there are sequences $\vec{c}=\left(c_{\nu}: \nu<\kappa\right)$ and $\vec{\beta}=\left(\beta_{\nu}: \nu<\kappa\right)$ such that $\vec{\beta}$ is unbounded in o( $\left.\alpha^{\prime}\right)$ and $\vec{c}$ is a $\kappa$-set of indiscernibles for $\vec{\beta}$.

Proof. Pick $N \prec H_{\kappa^{+}}$such that ${ }^{<\kappa} N \subset N$ and $\kappa+1 \cup\left\{\alpha^{\prime}\right\} \subset N$. By elementarity $N$ contains a sequence $(\vec{c}(\xi): \nu \in \kappa)$ of $\kappa$-sequences for sequences $\vec{\beta}(\xi)$, where $\bigcup_{\xi<\kappa} \bigcup_{\nu<\kappa} \beta(\xi)(\nu)=o\left(\alpha^{\prime}\right)$. We can take a diagonal sequence, $c_{\nu}=c(\nu)(f(\nu))$ for $\nu \in \kappa$, so that $\vec{c}=\left(c_{\nu}: \nu \in \kappa\right)$ is unbounded in $\kappa$ and $\vec{\beta}=(\beta(\nu)(f(\nu)): \nu<\kappa)$ is increasing and unbounded in $o\left(\alpha^{\prime}\right)$. We claim that $\vec{c}$ is a $\kappa$-set of indiscernibles for $\vec{\beta}$. If it is not then there is an $N^{\prime} \supset N$ containing a witness that it is not. Now each $\vec{c}(\xi)$, being a $\kappa$-set of indiscernibles for $\vec{\beta}(\xi)$, satisfies that $c(\xi)(\nu) \in C^{N^{\prime}}\left(\alpha^{\prime}, \beta(\xi)(\nu)\right)$ for sufficiently large $\nu$. By Lemma 1.5(iii) we can conclude that there is $\zeta<\alpha^{\prime}$ such that for all $\xi$ and $\nu$ with $c(\xi)(\nu)>\zeta$ we have $c \in C^{N^{\prime}}\left(\alpha^{\prime}, \beta(\xi)(\nu)\right)$, contradicting the assumption that $N^{\prime}$ contains a witness to the failure of $\vec{c}$ to be a $\kappa$-set of indiscernibles.

These lemmas enable us to analyze the indiscernibles from $j\left(i^{*}\right) j$ for ordinals $\alpha=j\left(\alpha^{\prime}\right)$ in $K(j(\mathcal{F}))$. If $\alpha^{\prime}$ has a $\kappa$-set of indiscernibles in $V$ for cofinally many measures, then using Lemma 5.2(ii) and (if $\operatorname{cf}(o(\alpha))=\kappa)$ Lemma 5.3 we see that $C^{j}$ has $j(\kappa)$-sets of indiscernibles for cofinally many measures on $j(\alpha)$ in $j(\mathcal{F})$ and these $j(\kappa)$-sets are in $V^{\kappa} / U$ so the map $j\left(i^{*}\right)$ does not do anything to $\alpha$. On the other hand if $\alpha^{\prime}$ does not have a $\kappa$-set of indiscernibles for cofinally many measures then, since for sufficiently large measures on $\alpha$ there are no $j(\kappa)$-sets of indiscernibles in $V^{\kappa} / U, j\left(i^{*}\right)$ will add them. By Lemma $5.1 C^{j}$ only has indiscernibles for boundedly many measures on $\alpha$, so the indiscernibles which are generated by $j$ can be absorbed by those added by $j\left(i^{*}\right)$. In either case we end up with exactly $j(\kappa)$-sets of indiscernibles for all measures on $\alpha$.

Now we turn to the ordinals in $K(\xi)$ which are not in the range of $j$. Each such ordinal $\alpha$ has the form $\alpha=[\lambda \nu \alpha(\nu)]$, where $\lambda \nu \alpha(\nu)$ is a strictly increasing function. In the following we assume that $\kappa+1 \cup\{\lambda \nu \alpha(\nu)\} \subset N$. If $\alpha$ is not itself an indiscernible, that is, if $\alpha \in j\left(g_{1}\right)^{\prime \prime} \alpha$, then we assume that $g_{1} \in N$. Otherwise if $\alpha \in C^{j}\left(\alpha^{\prime}, \beta^{\prime}\right)$ where $\alpha^{\prime}=\left[\lambda \nu \alpha^{\prime}(\nu)\right] \in j\left(g_{1}\right)^{\prime \prime} \alpha$ and $\beta^{\prime}=\left[\lambda \nu \beta^{\prime}(\nu)\right] \in j\left(g_{2}\right)^{\prime \prime} \alpha$ then we assume that $\left\{\lambda \nu \alpha^{\prime}(\nu), \lambda \nu \beta^{\prime}(\nu), g_{1}, g_{2}\right\} \subset N$. In this case when we write something of the form $\gamma \in j(g)^{\prime \prime} c$, for $\gamma \in o(\alpha)$, it is to be understood to mean $\exists \gamma^{\prime} \in o\left(\alpha^{\prime}\right)\left(\gamma^{\prime} \in j(g)^{\prime \prime} c\right.$ and $\left.\gamma=C\left(\alpha^{\prime}, \gamma^{\prime}, \beta^{\prime}\right)(\alpha)\right)$. In addition any indiscernibles $c$ mentioned will be assumed to be large enough that $\alpha^{\prime} \in j\left(g_{1}\right)^{\prime \prime} c$ and $\beta^{\prime} \in j\left(g_{2}\right)^{\prime \prime} c$. 
5.4. Proposition. Suppose that $c=[\lambda \nu c(\nu)] \in C^{j}(\alpha, \beta)$, where $\beta=$ $[\lambda \nu \beta(\nu)] \in j(g)^{\prime \prime} c$. Then $\left\{\nu: c(\nu) \in C^{N}(\alpha(\nu), \beta(\nu))\right\} \in U$.

PROOF. Exactly like the proof that $A \in U$ in the proof of Lemma 5.1.

5.5. Proposition. Suppose that $c(\nu) \in C^{N}(\alpha(\nu), \beta(\nu))$ for almost every $\nu$. Then $c=[\lambda \nu c(\nu)] \in C^{j}(\alpha, \beta)$ where $\beta=[\lambda \nu \beta(\nu)]$.

PROOF. If not then by Proposition 5.4 we can find $N^{\prime} \supset N$ such that $\{\nu: c(\nu) \notin$ $C^{N^{\prime}}(\alpha(\nu), \beta(\nu)\} \in U$, contradicting Lemma 1.2 which implies that this set is finite.

Thus indiscernibles in $C^{j}$ correspond directly to indiscernibles in $C^{N}$. We now will show that $j(\kappa)$-sets of indiscernibles both in $C^{j}$ and in $V^{\kappa} / U$ correspond to $\kappa$-sets of indiscernibles in $C^{N}$. This will show that, as before, either there is a $j(\kappa)$-set of indiscernibles in $C^{j}$ for cofinally many measures, in which case $V^{\kappa} / U$ also has such sets and $j\left(i^{*}\right)$ does nothing with $\alpha$, or else $C^{j}$ has something less than cofinally many $j(\kappa)$-sets of indiscernibles, in which case $j\left(i^{*}\right)$ adds such sets and they absorb whatever indiscernibles are in $C^{j}$. Thus the next two lemmas complete the proof of Lemma 4.11(i).

5.6. LEMMA. Suppose that in $\mathrm{C}^{j}$ there are $j(\kappa)$-sets of indiscernibles for cofnally many measures on $\alpha$. Then the set of $\nu$ such that there are $\kappa$-sets of indiscernibles for cofinally many measures on $\alpha(\nu)$ is in $U$.

Proof. Suppose that $\beta \leq o(\alpha)$ has cofinality $j(\kappa)$ and that $\alpha$ is an accumulation point for $\beta$ in $C^{j}$. Suppose that $\beta=[\lambda \nu \beta(\nu)] \in j(g)^{\prime \prime} c_{0}$, where $c_{0}=\left[\lambda \nu c_{0}(\nu)\right]$, and that $\left\{\lambda \nu \beta(\nu), g, \lambda \nu c_{0}(\nu)\right\} \subset N$. We will show that if $A=\{\nu \in \kappa: \alpha(\nu)$ is an accumulation point for $\beta(\nu)$ in $\left.C^{N}\right\}$ then $A \in U$. By Lemma 1.2 this will give a $\kappa$ set of indiscernibles for measures up to $\beta(\nu)$ in $\mathcal{F}$ and the conclusion of the lemma follows easily.

Suppose that $A \notin U$. Then for all $\nu \notin A$ there are $\xi(\nu)<\alpha(\nu)$ and $\gamma(\nu)<\beta(\nu)$ such that $C^{N}\left(\alpha(\nu), \xi^{\prime}\right) \cap[\xi(\nu), \alpha(\nu))=\varnothing$ for all $\xi^{\prime}$ with $\gamma(\nu) \leq \xi^{\prime}<\beta(\nu)$. Since $\alpha$ is an accumulation point for $C^{j}$ up to $\beta$, there are $c$ and $\delta$ with $c \in C^{j}(\alpha, \beta)$, $[\lambda \nu \xi(\nu)]<c<\alpha$, and $[\lambda \nu \gamma(\nu)]<\delta<\beta$. If $N^{\prime} \supset N$ with $\{\lambda \nu \delta(\nu), \lambda \nu c(\nu)\} \subset N^{\prime}$ then

$$
B=\left\{\nu \in \kappa \backslash A: c(\nu) \in C^{N^{\prime}}(\alpha(\nu), \delta(\nu))\right\} \in U .
$$

Let $D$ be an infinite subset of $B$. Then $N^{\prime}$ satisfies that for every set $g \in K(\mp)$ we have for all but finitely many $\nu \in D$ and for all $x \in g^{\prime \prime}(c(\nu))$ that $x \in \mathcal{F}(\alpha(\nu), \delta(\nu))$ iff $c(\nu) \in x$. Then $(c(\nu): \nu \in D)$ and $(\delta(\nu): \nu \in D)$ have the same property in $V$, and it follows that there are sequences $\left(c^{\prime}(\nu): \nu \in D\right)$ and $\left(\delta^{\prime}(\nu): \nu \in D\right)$ in $N$ with the same property such that $\xi(\nu) \leq c^{\prime}(\nu)<\alpha(\nu)$ and $\gamma(\nu) \leq \delta^{\prime}(\nu)<\beta(\nu)$. But then we must have $c^{\prime}(\nu) \in C^{N}\left(\alpha(\nu), \delta^{\prime}(\nu)\right)$ for all but finitely many $\nu$ 's, contradicting the definition of $D$.

5.7. LemmA. Suppose that $\{\beta(\nu): \nu \in \kappa\} \subset N$ and for almost every $\nu$ there are $\kappa$-sets of indiscernibles in $V$ for measures below $\beta(\nu)$. Then $\alpha(\nu)$ is an accumulation point for $\mp$ below $\beta(\nu)$ for almost every $\nu$.

ProOF. Let $A$ be the set of $\nu$ such that there is such a $\kappa$-set. Since there exist $\kappa$-sets of indiscernibles, such sets exist in $N$ : that is, for each $\nu \in A$ there are sequences $\left(c_{\xi}: \xi \in \kappa\right)$ and $\left(\beta_{\xi}: \xi \in \kappa\right)$ in $N$ such that $\beta_{\xi} \in j(g)^{\prime \prime} c_{\xi}$ for all $\xi<\kappa$ and 
some $g$ in $K(\mathcal{F}) \cap N$. It follows by $5.2(\mathrm{i})$ that $c_{\xi} \in C^{N}\left(\alpha(\nu), \beta_{\xi}\right)$ for all sufficiently large $\xi$.

PrOOF OF 4.11(ii). We need to show that if $c \in C^{i^{*}}$, the sequence of indiscernibles added by $i^{*}$, then $j(c)=j^{*}(c)$. We can define supports in $C^{i^{*}}$ as we did in Definition 4.5 for $C^{j}$, with the difference that in the case of $C^{i^{*}}$ we have more that $\omega$ accumulation points and hence need the extra parameter in $N$ : There are indiscernibles $\vec{c}$ such that $\delta=\sup (\vec{c})<c$ and $c=N\left(\alpha, \beta, \delta, \nu, \beta^{\prime}\right)$ where $\nu<\kappa$, $\left(\alpha, \beta, \beta^{\prime}\right)$ are definable for $C^{i^{*}}$ from $\vec{c}$, and there are no accumulation points $c^{\prime}$ for $\gamma>\beta$ such that $\delta<c^{\prime} \leq c$. We can assume as an induction hypothesis that $j^{*}(\vec{c})=j(\vec{c})$ and hence $j^{*}(\alpha)=j(\alpha), j^{*}(\beta)=j(\beta)$ and $j^{*}(\delta)=j(\delta)$. Since $\nu<\kappa$, $j^{*}(\nu)=j(\nu)=\nu$ and it will be enough to show that

$$
j(c)=N^{j i^{*}}\left(j(\alpha), j(\beta), j(\delta), \nu, j\left(\beta^{\prime}\right)\right)
$$

and

$$
j^{*}(c)=N^{j^{*} i^{*}}\left(j^{*}(\alpha), j^{*}(\beta), j^{*}(\delta), \nu, j^{*}\left(\beta^{\prime}\right)\right)
$$

This is easy to see for $j^{*}(c)$, since $j^{*}$ only adds indiscernibles after those added by $i^{*}$. For $j(c)$ we have by elementarity that

$$
j(c)=j\left(N^{i^{*}}\left(\alpha, \beta, \delta, \nu, \beta^{\prime}\right)\right)=j\left(N^{i^{*}}\right)\left(j(\alpha), j(\beta), j(\delta), \nu, j\left(\beta^{\prime}\right)\right) .
$$

Now $j\left(N^{i^{*}}\right)=N^{j\left(i^{*}\right)}$, that is, it only looks at the indiscernibles addded by $j\left(i^{*}\right)$ rather than all of those added by $j i^{*}=j\left(i^{*}\right) j$. Thus we need to see that there are no indiscernibles added by $j$ which would come in ahead of those added by $j\left(i^{*}\right)$ and thus make $N^{j\left(i^{*}\right) j}$ different from $N^{j\left(i^{*}\right)}$. If the measures below $\beta$ on $\alpha$ which have indiscernibles in $C^{j}$ larger that $j(\delta)$ are bounded in $\beta$ then there is no problem, since such indiscernibles will not affect the value of $N^{j\left(i^{*}\right) j}\left(j(\alpha), j(\beta), j(\delta), \nu, j\left(\beta^{\prime}\right)\right)$. If they are unbounded in $\beta$ then by Lemma 5.1 there are unboundedly many such measures with $\kappa$-sets of indiscernibles in $V$, in which case there was no need to use $i^{*}$ to add such sets: i.e., $c=N^{i^{*}}\left(\alpha, \beta, \delta, \nu, \beta^{\prime}\right)$ does not exist.

\section{REFERENCES}

[D-J81] A. J. Dodd and R. Jensen, The core model, Ann. Math. Logic 20 (1981), 43-75.

[D-J82a] _ , The covering lemma for K, Ann. Math. Logic 22 (1982), 1-30.

[D-J82b] _ , The covering lemma for $L(U)$, Ann. Math. Logic 22 (1982), 127-135.

[K-P71] K. Kunen and J. P. Paris, Boolean extensions and measurable cardinals, Ann. Math. Logic 2 (1971), 359-378.

[Ma78] M. Magidor, Changing cofinality of cardinals, Fund. Math. 99 (1978), 61-71.

[Mi74] W. Mitchell, Sets constructible from sequences of ultrafilters, J. Symbolic Logic 39 (1974), 57-66.

[Mi82] __ How weak is a closed unbounded ultrafilter, Logic Colloquium '80 (D. van Dalen, D. Lascar, J. Smiley, eds.), Studies in Logic, vol. 108, North-Holland, Amsterdam, 1982, pp. 209-230.

[Mi84a] _ The core model for sequences of measures. I, Math. Proc. Cambridge Philos. Soc. 95 (1984), 229-252.

[Mi84b] _ Indiscernibles, skies and ultrafilters, Contemporary Math. 31 (1984), 161-182. [Mi] _ The core model for sequences of measures. II (to appear).

[Pr71] K. Prikry, Changing measurable cardinals into accessible cardinals, Dissertationes Math. (Rozprawy Mat.) 68 (1971), 359-378.

Department of Mathematics, Pennsylvania State University, University PARK, PENNSYLVANIA 16802 